\title{
On the asymptotic distribution of the singular values of powers of random matrices
}

\author{
N. Alexeev \\ S.-Peterburg state University \\ S.-Peterburg, Russia
}

\author{
F. Götze \\ Faculty of Mathematics \\ University of Bielefeld \\ Germany
}

\author{
A. Tikhomirov ${ }^{1}$ \\ Department of Mathematics \\ Komi Research Center of Ural Branch of RAS \\ Syktyvkar, Russia
}

May 24, 2022

$\|$

\begin{abstract}
We consider powers of random matrices with independent entries. Let $X_{i j}, i, j \geq 1$, be independent complex random variables with $\mathbf{E} X_{i j}=0$ and $\mathbf{E}\left|X_{i j}\right|^{2}=1$ and let $\mathbf{X}$ denote an $n \times n$ matrix with $[\mathbf{X}]_{i j}=X_{i j}$, for $1 \leq i, j \leq n$. Denote by $s_{1}^{(m)} \geq \ldots \geq s_{n}^{(m)}$ the singular values of the random matrix $\mathbf{W}:=n^{-\frac{m}{2}} \mathbf{X}^{m}$ and define the empirical distribution of the squared singular values by

$$
\mathcal{F}_{n}^{(m)}(x)=\frac{1}{n} \sum_{k=1}^{n} I_{\left\{s_{k}^{(m) 2} \leq x\right\}},
$$

where $I_{\{B\}}$ denotes the indicator of an event $B$. We prove that under a Lindeberg condition for the fourth moment that the expected spectral distribution $F_{n}^{(m)}(x)=$ E $\mathcal{F}_{n}^{(m)}(x)$ converges to the distribution function $G^{(m)}(x)$ defined by its moments

$$
\alpha_{k}(m):=\int_{\mathbb{R}} x^{k} d G(x)=\frac{1}{m k+1}\left(\begin{array}{c}
k m+k \\
k
\end{array}\right) .
$$

\footnotetext{
${ }^{1}$ Partially supported by RFBF grant N 09-01-12180. Partially supported RFBR-DFG, grant N 09-0191331. Partially supported by CRC 701 "Spectral Structures and Topological Methods in Mathematics", Bielefeld
} 


\section{Introduction}

Let $X_{i j}, i, j \geq 1$, be independent complex random variables with $\mathbf{E} X_{i j}=0$ and $\mathbf{E}\left|X_{i j}\right|^{2}=$ 1 and $\mathbf{X}$ is an $n \times n$ matrix with $[\mathbf{X}]_{i j}=X_{i j}$, for $1 \leq i, j \leq n$. Denote by $s_{1}^{(m)} \geq \ldots \geq$ $s_{n}^{(m)}$ the singular values of the random matrix $\mathbf{W}:=n^{-\frac{m}{2}} \mathbf{X}^{m}$ and define the empirical distribution of its squared singular values by

$$
\mathcal{F}_{n}^{(m)}(x)=\frac{1}{n} \sum_{k=1}^{n} \mathbb{I}\left\{s_{k}^{(m)^{2}} \leq x\right\}
$$

where $\mathbb{I}\{B\}$ denotes the indicator of an event $B$. We shall investigate the convergence of the expected spectral distribution $F_{n}^{(m)}(x)=\mathbf{E} \mathcal{F}_{n}^{(m)}(x)$ to the distribution function $G^{(m)}(x)$ defined by its moments

$$
\alpha_{k}(m):=\int_{\mathbb{R}} x^{k} d G(x)=\frac{1}{m k+1}\left(\begin{array}{c}
k m+k \\
k
\end{array}\right) .
$$

The sequence $\alpha_{k}(m)$ consists of the so-called Fuss-Catalan Numbers. This sequence defines a distribution with Stieltjes transform $s^{(m)}(z)$ satisfying the equation (1.4) below. We consider the Kolmogorov distance between the distributions $F_{n}^{(m)}(x)$ and $G^{(m)}(x)$, that is

$$
\Delta_{n}^{(m)}:=\sup _{x}\left|F_{n}^{(m)}(x)-G^{(m)}(x)\right| .
$$

The main result of this paper is the following

Theorem 1.1. Let $\mathbf{E} X_{j k}=0, \mathbf{E}\left|X_{j k}\right|^{2}=1, \mathbf{E}\left|X_{j k}\right|^{4} \leq M<\infty$. Assume that for any $\tau>0$

$$
L_{n}(\tau):=\frac{1}{n^{2}} \sum_{j, k=1}^{n} \mathbf{E}\left|X_{j k}\right|^{4} I\left\{\left|X_{j k}\right|>\tau \sqrt{n}\right\} \rightarrow 0 \quad \text { as } n \rightarrow \infty,
$$

where $I\{E\}$ denotes indicator of an event $E$. Then, for any fixed $m \geq 2$,

$$
\lim _{n \rightarrow \infty} \sup _{x}\left|F_{n}^{(m)}(x)-G^{(m)}(x)\right|=0 .
$$

Corollary 1.1. Let $X_{j k}$ are independent identically distributed complex random variables. Let

$$
\mathbf{E} X_{j k}=0, \quad \mathbf{E}\left|X_{j k}\right|^{2}=1, \quad \mathbf{E}\left|X_{j k}\right|^{4}=M<\infty
$$

Then, for any fixed $m \geq 2$,

$$
\lim _{n \rightarrow \infty} \Delta_{n}^{(m)}=0 .
$$

Oravecz in 2001, [6], studied the so called $\mathcal{R}$-elements introduced by Voiculescu and has shown that the $m$-th powers of these elements have a distribution whose moments are Fuss-Catalan numbers. These numbers satisfy the following simple recurrence relation

$$
\alpha_{k}(m)=\sum_{k_{0}+\cdots+k_{m}=k-1} \prod_{\nu=0}^{m} \alpha_{k_{\nu}}(m) .
$$


Denote by $s^{(m)}(z)$ the Stieltjes transform of the distribution with moments $\alpha_{k}(m)$. Using equality (1.3), we may show that the Stieltjes transform $s^{(m)}(z)$ satisfies the equation

$$
1+z s^{(m)}(z)+(-1)^{m+1} z^{m}\left(s^{(m)}(z)\right)^{m+1}=0 .
$$

Distributions with such a Stieltjes transform belong to the class of the so-called Free Bessel Laws which are described in Banica and others [3. This distribution has been studied also in [7]. Using Free probability theory it is possible to prove the result of Theorem 1.1 for random matrices with independent entries, provided that all moments of $\mathbf{X}$ are finite. See for instance, Mingo and Speicher [7], and T. Banica and others [3. Theorem 1.1] was formulate in [2]. In [1] we gave a proof of Theorem 1.1 by the method of moments. Here we present a proof of Theorem 1.1 using Stieltjes transforms. This approach allows us to get some bound of the rate of convergence. Our proof of Theorem 1.1 is based on the representation (1.4). We shall investigate the Stieltjes transform $s_{n}^{(m)}(z)$ of the distribution function $F_{n}^{(m)}(x)$ and we shall show that $s_{n}^{(m)}(z)$ satisfies an equation

$$
1+z s_{n}^{(m)}(z)+(-1)^{m+1} z^{m}\left(s_{n}^{(m)}(z)\right)^{m+1}=\delta_{n}(z)
$$

with some function $\delta_{n}(z) \rightarrow 0$ as $n \rightarrow \infty$. From these two relations we get that $s_{n}^{(m)}(z)$ converges to $s^{(m)}(z)$ uniformly on any compact set in the upper half-plane $\mathcal{K} \subset \mathcal{C}^{+}$. The last claim is equivalent to weak convergence of the distribution functions $F_{n}^{(m)}(x)$ to the distribution function $F^{(m)}(x)$.

\section{Auxiliary results}

In this Section we describe a symmetrization of one-sided distributions and a special representation of the symmetrizing distribution of squared singular values of random matrices. Furthermore, we shall modify the random matrix $X$ by truncation of its entries. By condition (1.2), we get $\tau^{-q} L_{n}(\tau) \rightarrow 0$ ), for any $\tau>0$ and that for any $q>0$ the function $\tau^{-q} L_{n}(\tau)$ is not increasing in $\tau$. This implies that we may choose a sequence of positive numbers $\tau_{n}>0, n=1,2 \ldots$ such that

$$
\tau_{n} \rightarrow 0, \quad \text { and } \quad L_{n}(\tau) \leq \tau_{n}^{6}, \quad \text { as } \quad n \rightarrow \infty
$$

\subsection{Truncation}

We call the matrix $\widetilde{\mathbf{X}}$ the truncation of $\mathbf{X}$ if

$$
\widetilde{X}_{i j}=\left\{\begin{array}{l}
X_{i j}, \text { if }\left|X_{i j}\right|<\tau_{n} \sqrt{n} \\
0, \text { otherwise }
\end{array} .\right.
$$

Denote by $\widetilde{s}_{1}^{(m)} \geq \ldots \geq \widetilde{s}_{n}^{(m)}$ the singular values of the random matrix $\widetilde{\mathbf{W}}:=n^{-\frac{m}{2}} \widetilde{\mathbf{X}}^{m}$ and define the empirical distribution of its squared singular values (eigenvalues of the matrix 
$\left.\widetilde{\mathbf{V}}=\widetilde{\mathbf{W}} \widetilde{\mathbf{W}}^{*}\right)$ by $\widetilde{\mathcal{F}}_{n}^{(m)}(x)=\frac{1}{n} \sum_{k=1}^{n} I\left\{\left(\widetilde{s}_{k}^{(m)}\right)^{2} \leq x\right\}$. Let $\widetilde{F}_{n}^{(m)}(x)=\mathbf{E} \widetilde{\mathcal{F}}_{n}^{(m)}(x)$. It is straightforward to check that

$$
\begin{aligned}
\sup _{x}\left|\widetilde{F}_{n}^{(m)}(x)-F_{n}^{(m)}(x)\right| & \leq \sum_{j, k=1}^{n} \operatorname{Pr}\left\{\left|X_{j k}\right| \geq c n^{\frac{1}{2}}\right\} \\
& \leq \frac{1}{n^{2} \tau_{n}^{4}} \sum_{j, k=1}^{n} \mathbf{E}\left|X_{j k}\right|^{4} I\left\{\left|X_{j k}\right|>\tau_{n} \sqrt{n}\right\}=\frac{L_{n}\left(\tau_{n}\right)}{\tau_{n}^{4}} \leq \tau_{n}^{2} .
\end{aligned}
$$

Introduce the matrices $\widehat{\mathbf{X}}:=\widetilde{\mathbf{X}}-\mathbf{E} \widetilde{\mathbf{X}}$ and $\widehat{\mathbf{W}}=\widehat{\mathbf{X}}^{m}$ and $\widehat{\mathbf{V}}=\widehat{\mathbf{W}} \widehat{\mathbf{W}}^{*}$. Let $\widehat{\mathcal{F}}_{n}^{(m)}$ by the empirical distribution of its eigenvalues (squared singular values of $\widehat{\mathbf{W}}$ ) and $\widehat{F}_{n}^{(m)}=\mathbf{E} \widehat{\mathcal{F}}_{n}^{(m)}$. Let $\widehat{s}_{n}^{(m)}(z)$ denote the Stieltjes transform of $\widehat{F}_{n}^{(m)}$. Introduce the resolvent matrices

$$
\widehat{\mathbf{R}}=(\widehat{\mathbf{V}}-z \mathbf{I})^{-1}, \quad \text { and } \quad \widetilde{\mathbf{R}}=(\widetilde{\mathbf{V}}-z \mathbf{I})^{-1}
$$

We have

$$
\widetilde{s}_{n}^{(m)}(z)=\frac{1}{n} \mathbf{E} \operatorname{Tr} \widetilde{\mathbf{R}} \text { and } \quad \widehat{s}_{n}(z)=\frac{1}{n} \mathbf{E} \operatorname{Tr} \widehat{\mathbf{R}} .
$$

Applying the resolvent equality

$$
(\mathbf{A}+\mathbf{B}-z \mathbf{I})^{-1}=(\mathbf{A}-z \mathbf{I})^{-1}-(\mathbf{A}-z \mathbf{I})^{-1} \mathbf{B}(\mathbf{A}+\mathbf{B}-z \mathbf{I})^{-1},
$$

we get

$$
\left|\widetilde{s}_{n}^{(m)}(z)-\widehat{s}_{n}^{(m)}(z)\right| \leq \frac{1}{n} \mathbf{E}|\operatorname{Tr} \widetilde{\mathbf{R}}(\widetilde{\mathbf{V}}-\widehat{\mathbf{V}}) \widehat{\mathbf{R}}|
$$

Using that $\operatorname{Tr} \widetilde{\mathbf{R}}(\widetilde{\mathbf{V}}-\widehat{\mathbf{V}}) \widehat{\mathbf{R}}=\operatorname{Tr}(\widetilde{\mathbf{V}}-\widehat{\mathbf{V}}) \widehat{\mathbf{R}} \widetilde{\mathbf{R}}$ and applying Hölder's inequality, we obtain

$$
\left|\widetilde{s}_{n}^{(m)}(z)-\widehat{s}_{n}^{(m)}(z)\right| \leq \frac{1}{\sqrt{n} v^{2}} \mathbf{E}^{\frac{1}{2}}\|\widehat{\mathbf{W}}-\widetilde{\mathbf{W}}\|_{2}^{2}\left(\mathbf{E}^{\frac{1}{2}}\|\widetilde{\mathbf{W}}\|^{2}+\mathbf{E}^{\frac{1}{2}}\|\widehat{\mathbf{W}}\|^{2}\right) .
$$

By definition of the matrices $\widetilde{\mathbf{W}}$ and $\widehat{\mathbf{W}}$, we get

$$
\widetilde{\mathbf{W}}-\widehat{\mathbf{W}}=\sum_{\nu=0}^{m-1} \widehat{\mathbf{X}}^{\nu}(\widetilde{\mathbf{X}}-\widehat{\mathbf{X}}) \widehat{\mathbf{X}}^{m-1-\nu}
$$

This implies that

$$
\|\widehat{\mathbf{W}}-\widetilde{\mathbf{W}}\|_{2}^{2} \leq m \sum_{\nu=0}^{m-1}\|\widetilde{\mathbf{X}}-\widehat{\mathbf{X}}\|_{2}^{2}\left\|\widehat{\mathbf{X}}^{\nu} \widetilde{\mathbf{X}}^{m-1-\nu}\right\|_{2}^{2} .
$$

Applying Lemma 5.1, we obtain

$$
\mathbf{E}\|\widehat{\mathbf{W}}-\widetilde{\mathbf{W}}\|_{2}^{2} \leq C n\|\mathbf{E} \widetilde{\mathbf{X}}\|_{2}^{2} \leq \frac{C M}{n \tau_{n}^{6}} L_{n}\left(\tau_{n}\right) \leq C n^{-1} .
$$


Inequalities (2.11) and (2.8) together imply

$$
\left|\widehat{s}_{n}^{(m)}(z)-\widehat{s}_{n}^{(m)}(z)\right| \leq \frac{C}{\sqrt{n} v^{2}}
$$

Now we conclude that

$$
\lim _{n \rightarrow \infty} \sup _{x}\left|F_{n}^{(m)}-G^{(m)}(x)\right|=\lim _{n \rightarrow \infty} \sup _{x}\left|\mathbf{E} \widehat{F}_{n}^{(m)}-G^{(m)}(x)\right| .
$$

In the what follows we may assume without lost of generality that

$$
\mathbf{E} X_{j k}=0, \quad \mathbf{E}\left|X_{j k}\right|^{2}=1, \quad \text { and } \quad\left|X_{j k}\right| \leq \tau_{n} \sqrt{n},
$$

for some $\tau_{n}>0$ such that $\tau_{n} \rightarrow 0, L_{n}\left(\tau_{n}\right) \leq \tau_{n}^{6}$ as $n \rightarrow \infty$.

\subsection{Symmetrization}

We shall use the following "symmetrization" of one-sided distributions. Let $\xi^{2}$ be a positive random variable with distribution function $F(x)$. Define $\widetilde{\xi}:=\varepsilon \xi$ where $\varepsilon$ a Rademacher random variable with $\operatorname{Pr}\{\varepsilon= \pm 1\}=1 / 2$ which is independent of $\xi$. Let $\widetilde{F}(x)$ denote the distribution function of $\widetilde{\xi}$. It satisfies the equation

$$
\widetilde{F}(x)=1 / 2\left(1+\operatorname{sgn}\{x\} F\left(x^{2}\right)\right),
$$

We shall apply this symmetrization to the distribution of the squared singular values of the matrix W. Introduce the following matrices

$$
\mathbf{V}=\left(\begin{array}{cc}
\mathbf{W} & \mathbf{O} \\
\mathbf{O} & \mathbf{W}^{*}
\end{array}\right), \quad \mathbf{J}=\left(\begin{array}{cc}
\mathbf{O} & \mathbf{I}_{n} \\
\mathbf{I}_{n} & \mathbf{O}
\end{array}\right), \quad \text { and } \quad \widehat{\mathbf{V}}=\mathbf{V} \mathbf{J}
$$

Here and in the what follows $\mathbf{A}^{*}$ denotes the adjoined (transposed and complex conjugate) matrix $\mathbf{A}$ and $\mathbf{I}_{k}$ denotes the unit matrix of order $k$. Note that $\widehat{\mathbf{V}}$ is a Hermitian matrix. The eigenvalues of the matrix $\widehat{\mathbf{V}}$ are $-s_{1}, \ldots,-s_{n}, s_{n}, \ldots, s_{1}$. Note that the symmetrization of the distribution function $\mathcal{F}_{n}(x)$ is a function $\widetilde{\mathcal{F}}_{n}(x)$ which is the empirical distribution function of the eigenvalues of the matrix $\widehat{\mathbf{V}}$. By (2.14), we have

$$
\Delta_{n}^{(m)}=\sup _{x}\left|\widetilde{F}_{n}^{(m)}(x)-\widetilde{G}^{(m)}(x)\right|,
$$

where $\widetilde{F}_{n}^{(m)}(x)=\mathbf{E} \widetilde{\mathcal{F}}_{n}(x)$ and $\widetilde{G}^{(m)}(x)$ denotes the symmetrization of the distribution function $G^{(m)}(x)$. Let $s(z)$ denote the Stieltjes transform of the random variable $\xi^{2}$ and let $\widetilde{s}(z)$ denote the Stieltjes transform of $\widetilde{\xi}$. Then

$$
\widetilde{s}(z)=z s\left(z^{2}\right) .
$$

Equations (1.4) and (2.16) together imply

$$
1+z \widetilde{s}^{(m)}(z)+(-1)^{m+1} z^{m-1}\left(\widetilde{s}^{(m)}(z)\right)^{m+1}=0 .
$$


In the what follows we shall consider the symmetrization of the distribution $F_{n}^{(m)}(x)$ and the Stieltjes transform of $\widetilde{F}_{n}^{(m)}(x)$. We shall omit " " in the notation of the distribution function $\widetilde{F}_{n}^{(m)}(x)\left(\widetilde{G}^{(m)}(x)\right)$ and the Stieltjes transform $\widetilde{s}_{n}^{(m)}(z a)\left(\widetilde{s}^{(m)}(z)\right)$. By $C$ (with an index or without it) we shall denote generic absolute constants, whereas $C(\cdot, \cdot)$ will denote positive constants depending on arguments. For every matrix $\mathbf{A}$ by $\|\mathbf{A}\|_{2}$ we shall denote the Hilbert-Schmidt norm of the matrix $\mathbf{A}$ and by $\|\mathbf{A}\|$ we shall denote the operator norm of the matrix $\mathbf{A}$.

\section{The proof of the main result for $m=2$}

First, we prove Theorem 1.1 for $m=2$. Introduce the matrices $\mathbf{H}$ and $\mathbf{J}$ by the equalities

$$
\mathbf{H}=\left(\begin{array}{cc}
\mathbf{X} & \mathbf{O} \\
\mathbf{O} & \mathbf{X}^{*}
\end{array}\right), \quad \mathbf{J}:=\left(\begin{array}{cc}
\mathbf{O} & \mathbf{I} \\
\mathbf{I} & \mathbf{O}
\end{array}\right) .
$$

Let $\mathbf{V}:=\mathbf{H}^{m} \mathbf{J}$, and $\mathbf{R}(z)$ denote the resolvent matrix of $\mathbf{V}$,

$$
\mathbf{R}(z):=(\mathbf{V}-z \mathbf{I})^{-1} .
$$

Furthermore, we note that the symmetrization of the distribution function $G_{2}(x)$ has a Stieltjes transform $s(z)$ which satisfies the following equation

$$
1+z s(z)-z s^{3}(z)=0 .
$$

We shall prove that in the case $m=2$ the Stieltjes transform of the expected spectral distribution function $s_{n}(z)=\int_{-\infty}^{\infty} \frac{1}{x-z} \mathrm{~d} F_{n}(x)$ satisfies the equation

$$
1+z s(z)-z s^{3}(z)=\delta_{n}(z)
$$

where $\delta_{n}(z)$ denotes some function such that $\delta_{n}(z) \rightarrow 0$ as $n \rightarrow \infty$. In the what follows we shall denote by $\varepsilon_{n}(z)$ a generic error term such that $\left|\varepsilon_{n}(z)\right| \leq C \tau_{n}^{a} v^{-b}$ for some positive constants $C, a$, and $b$.

We start from the obvious equality

$$
1+z s_{n}(z)=\frac{1}{2 n} \mathbf{E} \operatorname{Tr} \mathbf{V R}(z) .
$$

Using the definition of the matrices $\mathbf{V}, \mathbf{H}$ and $\mathbf{J}$, we get

$$
1+z s_{n}(z)=\frac{1}{2 n \sqrt{n}} \sum_{j, k=1}^{n} \mathbf{E} X_{j k}\left([\mathbf{H J R}]_{k j}+[\mathbf{H J R}]_{j+n, k+n}\right) .
$$

By Lemma 5.7 of the Appendix, we get

$$
\left.1+z s_{n}(z)\right)=\frac{1}{2 n \sqrt{n}} \sum_{j, k=1}^{n} \mathbf{E}\left[\frac{\partial \mathbf{H J R}}{\partial X_{j k}}\right]_{k j}+\frac{1}{2 n \sqrt{n}} \sum_{j, k=1}^{n} \mathbf{E}\left[\frac{\partial \mathbf{H J R}}{\partial X_{j k}}\right]_{j+n, k+n}+\varepsilon_{n}(z) .
$$


Let $\mathbf{e}_{1}, \ldots, \mathbf{e}_{2 n}$ be an orthonormal basic of $\mathbb{R}^{2 n}$. First we note that, for $1 \leq j, k \leq n$,

$$
\frac{\partial \mathbf{H}}{\partial X_{j k}}=\frac{1}{\sqrt{n}}\left(\mathbf{e}_{j} \mathbf{e}_{k}^{T}+\mathbf{e}_{k+n} \mathbf{e}_{j+n}^{T}\right)
$$

and

$$
\frac{\partial(\mathbf{H J})}{\partial X_{j k}}=\frac{1}{\sqrt{n}}\left(\mathbf{e}_{j} \mathbf{e}_{k+n}^{T}+\mathbf{e}_{k+n} \mathbf{e}_{j}^{T}\right) .
$$

Now we compute the derivatives of the resolvent matrix as follows

$$
\begin{aligned}
\frac{\partial \mathbf{R}}{\partial X_{j k}}= & -\frac{1}{\sqrt{n}} \mathbf{R}\left(\mathbf{e}_{j} \mathbf{e}_{k}^{T}+\mathbf{e}_{k+n} \mathbf{e}_{j+n}^{T}\right) \mathbf{H J R} \\
& -\frac{1}{\sqrt{n}} \mathbf{R H}\left(\mathbf{e}_{j} \mathbf{e}_{k+n}^{T}+\mathbf{e}_{k+n} \mathbf{e}_{j}^{T}\right) \mathbf{R} .
\end{aligned}
$$

and

$$
\begin{aligned}
\frac{\partial(\mathbf{H J R})}{\partial X_{j k}}=\frac{1}{\sqrt{n}}\left(\mathbf{e}_{j} \mathbf{e}_{k+n}^{T}+\mathbf{e}_{k+n} \mathbf{e}_{j}^{T}\right) \mathbf{R} & -\frac{1}{\sqrt{n}} \mathbf{H J R}\left(\mathbf{e}_{j} \mathbf{e}_{k}^{T}+\mathbf{e}_{k+n} \mathbf{e}_{j+n}^{T}\right) \mathbf{H J R} \\
& -\frac{1}{\sqrt{n}} \mathbf{H J R H}\left(\mathbf{e}_{j} \mathbf{e}_{k+n}^{T}+\mathbf{e}_{k+n} \mathbf{e}_{j}^{T}\right) \mathbf{R} .
\end{aligned}
$$

The equalities (3.5) and (3.9) together imply

$$
1+z s_{n}(z)=A_{1}+\cdots+A_{6}+\varepsilon_{n}(z)
$$

where

$$
\begin{aligned}
& A_{1}:=\frac{1}{2 n^{2}} \mathbf{E}\left(\sum_{j=1}^{n} \mathbf{R}_{j, j+n}+\sum_{j=1}^{n} \mathbf{R}_{j+n, j}\right), \\
& A_{2}:=-\frac{1}{2 n^{2}} \mathbf{E} \sum_{j, k=1}^{n}\left([\mathbf{H} J \mathbf{R}]_{j k}^{2}+[\mathbf{H J R}]_{j+n, k+n}^{2}\right), \\
& A_{3}:=-\frac{1}{n^{2}} \mathbf{E} \sum_{j=1}^{n}[\mathbf{H J R}]_{j, j+n} \sum_{k=1}^{n}[\mathbf{H J R}]_{k+n, k}, \\
& A_{4}:=-\frac{1}{2 n^{2}} \mathbf{E} \sum_{j, k=1}^{n}\left([\mathbf{H J R H}]_{k, j+n} \mathbf{R}_{k+n, j}+[\mathbf{H J R H}]_{j+n, k+n} \mathbf{R}_{j, k+n}\right), \\
& A_{5}:=-\frac{1}{2 n^{2}} \mathbf{E} \sum_{k=1}^{n}[\mathbf{H J R H}]_{k, k+n} \sum_{j=1}^{n} \mathbf{R}_{j j}, \\
& A_{6}:=-\frac{1}{2 n^{2}} \mathbf{E} \sum_{k=1}^{n}[\mathbf{H J R H}]_{k+n, k} \sum_{j=1}^{n} \mathbf{R}_{j+n, j+n} .
\end{aligned}
$$

We prove that the first four summands are negligible and the main asymptotic terms are the last two summands. We now start the investigation of these terms. 
Lemma 3.1. Under conditions of Theorem 1.1 we have

$$
\begin{aligned}
& \left|A_{5}+\left(\frac{1}{2 n} \sum_{k=1}^{n} \mathbf{E}[\mathbf{H J R H}]_{k, k+n}\right)\left(\frac{1}{n} \sum_{j=1}^{n} \mathbf{E} \mathbf{R}_{j j}\right)\right| \leq \frac{C}{n v^{2}}, \\
& \left|A_{6}+\left(\frac{1}{2 n} \sum_{k=1}^{n} \mathbf{E}[\mathbf{H J R H}]_{k+n, k}\right)\left(\frac{1}{n} \sum_{j=1}^{n} \mathbf{E} \mathbf{R}_{j+n, j+n}\right)\right| \leq \frac{C}{n v^{2}} .
\end{aligned}
$$

Proof. Using Cauchy's inequality we have

$$
\begin{aligned}
& \left|A_{5}+\left(\frac{1}{2 n} \sum_{k=1}^{n} \mathbf{E}[\mathbf{H J R H}]_{k, k+n}\right)\left(\frac{1}{n} \sum_{j=1}^{n} \mathbf{E} \mathbf{R}_{j j}\right)\right| \\
& \leq \mathbf{E}^{\frac{1}{2}}\left|\frac{1}{n}\left(\sum_{k=1}^{n}[\mathbf{H J R H}]_{k, k+n}-\mathbf{E} \sum_{k=1}^{n}[\mathbf{H J R H}]_{k, k+n}\right)\right|^{2} \mathbf{E} \frac{1}{2}\left|\frac{1}{n}\left(\sum_{j=1}^{n}\left(\mathbf{R}_{j j}-\mathbf{E} \mathbf{R}_{j j}\right)\right)\right|^{2} .
\end{aligned}
$$

Applying Lemma 5.4 with $p=2$ and $q=1$ and Lemma 5.3 (see the Appendix), we get

$$
\left|A_{5}+\left(\frac{1}{2 n} \sum_{k=1}^{n} \mathbf{E}[\mathbf{H J R H}]_{k, k+n}\right)\left(\frac{1}{n} \sum_{j=1}^{n} \mathbf{E} \mathbf{R}_{j j}\right)\right| \leq \frac{C}{n v^{2}} .
$$

Similar we prove the second inequality in (3.11). Thus the Lemma is proved.

Note that

$$
\frac{1}{n} \sum_{j=1}^{n} \mathbf{E} \mathbf{R}_{j j}=\frac{1}{n} \sum_{j=1}^{n} \mathbf{E} \mathbf{R}_{j+n, j+n}=s_{n}(z) .
$$

Lemma 3.1, equality (3.15) and the definition of matrix $\mathbf{H}$ together imply

$$
A_{5}=-s_{n}(z) \frac{1}{2 n} \sum_{j, k=1}^{n} \mathbf{E} X_{j k}[\mathbf{H J R}]_{j, k+n}+\frac{C \theta}{n v^{4}},
$$

and similarly

$$
A_{6}=-s_{n}(z) \frac{1}{2 n} \sum_{j, k=1}^{n} \mathbf{E} X_{j k}[\mathbf{H J R}]_{k+n, j}+\frac{C \theta}{n v^{4}},
$$

where $\theta$ denotes a quantity such that $|\theta| \leq 1$. Applying Lemma 5.7 and equalities (3.6)(3.9), we get

$$
\begin{aligned}
& A_{5}=-\frac{1}{2} s_{n}^{2}(z)+s_{n}^{2}(z) \frac{1}{2 n} \sum_{j=1}^{n} \mathbf{E}\left[\mathbf{H}^{2} \mathbf{J R}\right]_{j j}+A_{7}+A_{9} \\
& A_{6}=-\frac{1}{2} s_{n}^{2}(z)+s_{n}^{2}(z) \frac{1}{2 n} \sum_{j=1}^{n} \mathbf{E}\left[\mathbf{H}^{2} \mathbf{J R}\right]_{j+n, j+n}+A_{8}+A_{10}
\end{aligned}
$$


where

$$
\begin{aligned}
A_{7} & =s_{n}(z) \frac{1}{2 n^{2}} \sum_{j=1}^{n}[\mathbf{H J R}]_{j+n, j+n} \sum_{k=1}^{n}[\mathbf{H J R}]_{k+n, k}, \\
A_{8} & =s_{n}(z) \frac{1}{2 n^{2}} \sum_{j=1}^{n}[\mathbf{H J R}]_{j, j+n} \sum_{k=1}^{n}[\mathbf{H J R}]_{k, k}, \\
A_{9} & =s_{n}(z) \frac{1}{2 n^{2}} \sum_{j, k=1}^{n}[\mathbf{H J R H}]_{j+n, k+n} \mathbf{R}_{j, k+n} \\
A_{10} & =s_{n}(z) \frac{1}{2 n^{2}} \sum_{j, k=1}^{n}[\mathbf{H J R H}]_{j+n, k+n} \mathbf{R}_{j, k+n}
\end{aligned}
$$

By resolvent equality $\mathbf{I}+z \mathbf{R}=\mathbf{W R}$, we have

$$
\frac{1}{2 n}\left(\sum_{j=1}^{n} \mathbf{E}\left[\mathbf{H}^{2} \mathbf{J R}\right]_{j j}+\sum_{j=1}^{n} \mathbf{E}\left[\mathbf{H}^{2} \mathbf{J R}\right]_{j+n, j+n}\right)=1+z s_{n}(z) .
$$

Equalities (3.4), (3.18) and (3.19) together imply

$$
A_{5}+A_{6}=z s_{n}^{3}(z)+A_{7}+\cdots+A_{10} .
$$

Lemma 3.2. Under the conditions of Theorem 1.1 we have

$$
\max \left\{\left|A_{1}\right|,\left|A_{2}\right|,\left|A_{9}\right|,\left|A_{10}\right|,\left|A_{4}\right|\right\} \leq \frac{C}{n v^{2}} .
$$

Proof. We shall describe the estimate (3.21) for the quantity $A_{9}$ only. The other bounds will be similar. By Hölder's inequality, we have

$$
\left|A_{9}\right| \leq \frac{1}{n^{2}} \mathbf{E}\|\mathbf{H J R H}\|_{2}\|\mathbf{R}\|_{2} \leq \frac{1}{n^{\frac{3}{2}} v} \mathbf{E}\|\mathbf{H J R H}\|_{2} \|
$$

where $\|\cdot\|_{2}$ denotes the Hilbert-Schmidt norm of a matrix. Using

$$
\|\mathbf{H J R H}\|_{2}=\left\|\mathbf{H}^{2} \mathbf{J R}\right\|_{2} \leq\left\|\mathbf{H}^{2}\right\|_{2}\|\mathbf{R}\| \leq \frac{1}{v}\left\|\mathbf{H}^{2}\right\|_{2},
$$

and Lemma 5.1, we get

$$
\left|A_{9}\right| \leq \frac{C \sqrt{n}}{n^{\frac{3}{2}} v^{2}} \leq \frac{C}{n v^{2}}
$$

Thus the Lemma is proved.

Introduce the notations

$$
\begin{aligned}
& A:=\frac{1}{n} \sum_{j=1}^{n} \mathbf{E}[\mathbf{H J R}]_{j j}, \quad B:=\frac{1}{n} \sum_{j=1}^{n} \mathbf{E}[\mathbf{H J R}]_{j+n, j+n}, \\
& C:=\frac{1}{n} \sum_{j=1}^{n} \mathbf{E}[\mathbf{H J R}]_{j, j+n}, \quad D:=\frac{1}{n} \sum_{j=1}^{n} \mathbf{E}[\mathbf{H J R}]_{j+n, j}, \quad t(z):=\sum_{j=1}^{n} \mathbf{E} \mathbf{R}_{j, j+n} .
\end{aligned}
$$

Using these notations we prove the following 
Lemma 3.3. The following representations hold

$$
\begin{array}{ll}
A=-s_{n}(z) C-s_{n}(z) D+\varepsilon_{n}(z), & B=-s_{n}(z) D-s_{n}(z) C+\varepsilon_{n}(z), \\
C=-t_{n}(z) D-s_{n}(z) A+\varepsilon_{n}(z), & D=-t_{n}(z) C-s_{n}(z) B+\varepsilon_{n}(z),
\end{array}
$$

where $\left|\varepsilon_{n}(z)\right| \leq \frac{C \tau_{n}}{n v^{4}}$.

Proof. We start with the first equality. By definition of $A$, we have

$$
A=\frac{1}{n \sqrt{n}} \sum_{j, k=1}^{n} \mathbf{E} X_{j k} \mathbf{R}_{k+n, j} .
$$

Using Lemma 5.7, we get

$$
\begin{aligned}
A & =-\frac{1}{n^{2}} \sum_{j, k=1}^{n} \mathbf{E} \mathbf{R}_{j+n, j+n}[\mathbf{H J R}]_{k+n, k} \\
& -\frac{1}{n^{2}} \sum_{j, k=1}^{n} \mathbf{E}[\mathbf{R H}]_{j+n, j+n}[\mathbf{J R}]_{k+n, k}-\frac{1}{n^{2}} \sum_{j, k=1}^{n} \mathbf{E} \mathbf{R}_{j+n, k}[\mathbf{H J R}]_{j+n, k} \\
& -\frac{1}{n^{2}} \sum_{j, k=1}^{n} \mathbf{E}[\mathbf{R H}]_{k+n, j}[\mathbf{J R}]_{k, j}+\varepsilon_{n}(z) .
\end{aligned}
$$

Applying Lemma 5.4 and 5.5, we have

$$
A=-s_{n}(z) C-s_{n}(z) D+\varepsilon_{n}(z) .
$$

The proof of the other relations is similar.

We may write now

$$
A_{3}+A_{7}+A_{8}=-C D+\frac{1}{2} s_{n}(z)(B D+A C)=-\frac{1}{2}\left(C-s_{n}(z) B\right) D-\frac{1}{2}\left(D-s_{n}(z) A\right) C+\varepsilon_{n}(z) .
$$

Applying the results of Lemma 3.3. we obtain

$$
\begin{aligned}
A_{3}+A_{7}+A_{8} & =-\frac{1}{2}(D+C)^{2}-\frac{1}{2} t_{n}(z) C D+\varepsilon_{n}(z) \\
& =-\frac{1}{2}\left(1+\frac{t_{n}}{4}\right)(D+C)^{2}-\frac{1}{8} t_{n}(z)(C-D)^{2}+\varepsilon_{n}(z) \\
& =-\frac{\varepsilon_{n}^{2}(z)\left(1+t_{n}(z) / 4\right)^{2}}{2\left(1+t_{n}(z)-2 s_{n}^{2}(z)\right)^{2}}-\frac{\varepsilon_{n}^{2}(z)}{\left(1-t_{n}(z)\right)^{2}}+\varepsilon_{n}(z) .
\end{aligned}
$$

Consider first the case $v \geq 4$. Here we have

$$
\left|s_{n}(z)\right| \leq \frac{1}{4}, \quad\left|t_{n}(z)\right| \leq \frac{1}{4}
$$


These inequalities imply that for $v \geq 4$

$$
\left|A_{3}+A_{7}+A_{8}\right| \leq \frac{C \tau_{n}}{v^{4}}
$$

Inequalities (3.10), (3.20), (3.21), and (3.23) together imply

$$
1+z s_{n}(z)=z s_{n}^{3}(z)+\delta_{n}(z),
$$

where $\left|\delta_{n}(z)\right| \leq \frac{C \tau_{n}}{v^{4}}$, for $v>4$.

Lemma 3.4. Assuming the conditions of Theorem 1.1 there exists some positive constants $C_{0}, C_{1}$ such that, for $v \geq C_{0}$,

$$
\left|s(z)-s_{n}(z)\right| \leq \frac{C_{1}\left|\varepsilon_{n}(z)\right|}{v}
$$

Proof. First we note that

$$
\left|z s_{n}(z)\right| \leq 1+\frac{1}{v} \mathbf{E}^{\frac{1}{2}}\|\mathbf{V}\|_{2}^{2} .
$$

Applying Lemma 5.1 and that $\max \left\{|s(z)|, \mid s_{n}(z)\right\} \leq v^{-1}$, we get

$$
\max \left\{\left|z s_{n}^{2}(z)\right|,\left|z s(z) s_{n}(z)\right|\right\} \leq \frac{1}{v}\left(1+\frac{C}{v}\right) .
$$

Furthermore,

$$
\operatorname{Im}\left\{z s^{2}(z)\right\} \leq 0
$$

It follows from equality (1.4) that

$$
\operatorname{Im} z s^{2}(z)=\operatorname{Im}\left\{z+\frac{1}{s(z)}\right\}=\frac{v|s(z)|^{2}-\operatorname{Im} s(z)}{|s(z)|^{2}} .
$$

For a Stieltjes transform $t(z)$ of a random variable $\xi$ we have

$$
v|t(z)|^{2}-\operatorname{Im} t(z)=v\left(\left|\mathbf{E} \frac{1}{\xi-z}\right|^{2}-\mathbf{E}\left|\frac{1}{\xi-z}\right|^{2}\right) \leq 0 .
$$

Equalities (3.29) and (3.30) together imply (3.28). From relations (1.4) and (3.24) we obtain

$$
\left|s_{n}(z)-s(z)\right| \leq \frac{\left|\delta_{n}(z)\right|}{\left|z-z s^{2}(z)-z s(z) s_{n}(z)-z s_{n}^{2}(z)\right|} .
$$

Inequalities (3.27), (3.28) together imply that, for $v \geq 4 C$,

$$
\left|z-z s^{2}(z)-z s(z) s_{n}(z)-z s_{n}^{2}(z)\right| \geq \operatorname{Im}\left\{z-z s^{2}(z)-z s(z) s_{n}(z)-z s_{n}^{2}(z)\right\} \geq \frac{v}{2} .
$$

Inequalities (3.31) and (3.32) together completed the proof of lemma.

The last Lemma implies that there exists an open set in $\mathcal{C}^{+}$with non-empty interior such that $s_{n}(z)$ convergence to $s(z)$ on this set. The Stieltjes transform of these random variables is an analytic function on $\mathcal{C}^{+}$and locally bounded, that is $\left(\left|s_{n}(z)\right| \leq v^{-1}\right.$ for any $v>0$ ). By Montel's Theorem (see, for instance, [4], p. 153, Theorem 2.9) $s_{n}(z)$ converges to $s(z)$ uniformly on any compact set in the upper half-plane $\mathcal{K} \subset \mathcal{C}^{+}$. This implies that $\Delta_{n} \rightarrow 0$ as $n \rightarrow \infty$. Thus the proof of Theorem 1.1 in the case $m=2$ is complete. 


\section{The proof of the main result in general case}

Recall that $\mathbf{H}$ and $\mathbf{J}$ are defined by the equalities

$$
\mathbf{H}=\left(\begin{array}{cc}
\mathbf{X} & \mathbf{O} \\
\mathbf{O} & \mathbf{X}^{*}
\end{array}\right), \quad \mathbf{J}=\left(\begin{array}{cc}
\mathbf{O} & \mathbf{I} \\
\mathbf{I} & \mathbf{O}
\end{array}\right)
$$

Let $\mathbf{V}:=\mathbf{H}^{m} \mathbf{J}$, and $\mathbf{R}(z)$ denote the resolvent matrix of the matrix $\mathbf{V}$,

$$
\mathbf{R}(z):=(\mathbf{V}-z \mathbf{I})^{-1} .
$$

We shall use the following "symmetrization" of a one-sided distribution. Let $\xi^{2}$ be a positive random variable. Define $\widetilde{\xi}:=\varepsilon \xi$, where $\varepsilon$ denotes a Rademacher random variable with $\operatorname{Pr}\{\varepsilon= \pm 1\}=1 / 2$ which is independent of $\xi$. We apply this symmetrization to the distribution of the singular values of the matrix $\mathbf{X}^{2}$. Note that the symmetrized distribution function $\widetilde{F}_{n}(x)$ satisfies the equation

$$
\widetilde{F}_{n}(x)=1 / 2\left(1+\operatorname{sgn}\{x\} F_{n}\left(x^{2}\right)\right),
$$

and that this function is the empirical spectral distribution function of the random matrix

$$
\mathbf{V}=\left(\begin{array}{cc}
\mathbf{O} & \mathbf{X}^{m} \\
\mathbf{X}^{* m} & \mathbf{O}
\end{array}\right)
$$

Furthermore, note that the symmetrization of the distribution function $G(x)$ has the Stieltjes transform $s(z)$ which satisfies the following equation

$$
1+z s(z)+(-1)^{m+1} z^{m-1} s^{m+1}(z)=0 .
$$

In the rest of paper we shall prove that the Stieltjes transform of the expected spectral distribution function $s_{n}(z)=\int_{-\infty}^{\infty} \frac{1}{x-z} \mathrm{~d} \mathbf{E} \widetilde{F}_{n}(x)$ satisfies the equation

$$
1+z s_{n}(z)+(-1)^{m+1} z^{m-1} s_{n}^{m+1}(z)=\delta_{n}(z),
$$

where $\delta_{n}(z)$ denotes some remainder function such that $\delta_{n}(z) \rightarrow 0$ as $n \rightarrow \infty$.

We start from the obvious equality

$$
1+z s_{n}(z)=\frac{1}{2 n} \operatorname{Tr} \mathbf{V R}(z)
$$

Using the definition of the matrices $\mathbf{V}, \mathbf{H}$ and $\mathbf{J}$, we get

$$
1+z s_{n}(z)=\frac{1}{2 n \sqrt{n}} \sum_{j, k=1}^{n} \mathbf{E} X_{j k}\left(\left[\mathbf{H}^{m-1} \mathbf{J R}\right]_{k j}+\left[\mathbf{H}^{m-1} \mathbf{J R}\right]_{j+n, k+n}\right) .
$$

In order to simplify the calculations we shall assume that $X_{j k}$ are i.i.d. Gaussian random variables, and shall use the following well-known equality for a Gaussian r.v. $\xi$

$$
\mathbf{E} \xi f(\xi)=\mathbf{E} f^{\prime}(\xi)
$$


which holds for arbitrary differentiable functions $f(x)$, such that both sides are defined. By Lemma 5.7, we obtain that the error of the replacement by Gaussian r.v is of order $O\left(\tau_{n}\right)$. In the what follows we shall use the notation $\varepsilon_{n}(z)$ for functions satisfying $\left|\varepsilon_{n}(z)\right| \leq$ $C \tau_{n}^{a} v^{-b}$, for some positive constants $a, b$, and $C$. Let $\mathbf{e}_{1}, \ldots, \mathbf{e}_{2 n}$ denote an orthonormal basis of $\mathbb{R}^{2 n}$. First we note that

$$
\frac{\partial \mathbf{H}}{\partial X_{j k}}=\frac{1}{\sqrt{n}}\left(\mathbf{e}_{j} \mathbf{e}_{k}^{T}+\mathbf{e}_{k+n} \mathbf{e}_{j+n}^{T}\right)
$$

Now we may write the equality for the derivatives of the matrix $\mathbf{H}^{m-1} \mathbf{J R}$ as follows

$$
\begin{aligned}
\frac{\partial \mathbf{H}^{m-1} \mathbf{J R}}{\partial X_{j k}} & =\frac{1}{\sqrt{n}} \sum_{q=0}^{m-2} \mathbf{H}^{q}\left(\mathbf{e}_{j} \mathbf{e}_{k}^{T}+\mathbf{e}_{k+n} \mathbf{e}_{j+n}^{T}\right) \mathbf{H}^{m-2-q} \mathbf{J R} \\
& -\frac{1}{\sqrt{n}} \sum_{q=0}^{m-1} \mathbf{H}^{m-1} \mathbf{J} \mathbf{R} \mathbf{H}^{q}\left(\mathbf{e}_{j} \mathbf{e}_{k}^{T}+\mathbf{e}_{k+n} \mathbf{e}_{j+n}^{T}\right) \mathbf{H}^{m-1-q} \mathbf{J R} .
\end{aligned}
$$

The equalities (4.5) and (4.8) together imply

$$
1+z s_{n}(z)=A_{1}+A_{2}+B_{1}+B_{2}+C_{1}+C_{2}+D_{1}+D_{2}+\varepsilon_{n}(z),
$$

where

$$
\begin{aligned}
& A_{1}:=\sum_{q=0}^{m-2} \frac{1}{2 n^{2}} \mathbf{E} \sum_{j, k=1}^{n} \mathbf{H}_{k j}^{q}\left[\mathbf{H}^{m-2-q} \mathbf{J R}\right]_{k j}, \\
& A_{2}= \sum_{q=0}^{m-2} \frac{1}{2 n^{2}} \mathbf{E} \sum_{j, k=1}^{n} \mathbf{H}_{k, k+n}^{q}\left[\mathbf{H}^{m-2-q} \mathbf{J R}\right]_{j+n, j}, \\
& B_{1}:=-\sum_{q=0}^{m-1} \frac{1}{2 n^{2}} \mathbf{E} \sum_{j, k=1}^{n}\left[\mathbf{H}^{m-1} \mathbf{J R} \mathbf{H}^{q}\right]_{k, j}\left[\mathbf{H}^{m-1-q} \mathbf{J R}\right]_{k, j} \\
& B_{2}=-\sum_{q=0}^{m-1} \sum_{k, j=1}^{n} \mathbf{E}\left[\mathbf{H}^{m-1} \mathbf{J R} \mathbf{H}^{m-1-q}\right]_{k, k+n}\left[\mathbf{H}^{m-1-q} \mathbf{J R}\right]_{j+n, j}, \\
& C_{1}= \sum_{q=0}^{m-2} \frac{1}{2 n^{2}} \mathbf{E} \mathbf{H}_{j+n, k+n}^{q}\left[\mathbf{H}^{m-1-q} \mathbf{J R}\right]_{j+n, k+n}, \\
& C_{2}:= \sum_{q=0}^{m-2} \frac{1}{2 n^{2}} \mathbf{E} \sum_{j, k=1}^{n} \mathbf{H}_{j+n, j}^{q}\left[\mathbf{H}^{m-2-q} \mathbf{J R}\right]_{k, k+n} \\
& D_{1}:=-\sum_{q=0}^{m-1} \frac{1}{2 n^{2}} \mathbf{E} \sum_{j, k=1}^{n}\left[\mathbf{H}^{m-1} \mathbf{J R H}^{q}\right]_{j+n, k+n}\left[\mathbf{H}^{m-1-q} \mathbf{J R}\right]_{j+n, k+n} \\
& D_{2}=-\sum_{q=0}^{m-1} \sum_{k, j=1}^{n} \mathbf{E}\left[\mathbf{H}^{m-1} \mathbf{J R H}^{m-1-q}\right]_{j+n, j}\left[\mathbf{H}^{m-1-q} \mathbf{J R}\right]_{k, k+n} .
\end{aligned}
$$


Lemma 4.1. Under the conditions of Theorem 1.1 there exists a constant $C>0$ that the following inequality holds

$$
\max \left\{\left|A_{1}\right|,\left|B_{1}\right|\left|C_{1}\right|,\left|D_{1}\right|\right\} \leq \frac{C}{n v} .
$$

Proof. To prove this lemma it is enough to use Hölder's inequality and Lemma 5.1 in the Appendix.

Lemma 4.2. Under the conditions of Theorem 1.1 we have

$$
A_{2}=C_{2}=0 \text {. }
$$

Proof. The claim follows immediately from the equality $\mathbf{H}_{j, j+n}^{q}=0$.

To investigate the asymptotic behavior of $B_{2}$ and $D_{2}$ we introduce the notations

$$
\begin{aligned}
f_{\alpha, \beta} & :=\frac{1}{n} \sum_{j=1}^{n} \mathbf{E}\left[\mathbf{H}^{\alpha} \mathbf{J R H}\right]_{j, j+n}, \quad g_{\alpha, \beta}:=\frac{1}{n} \sum_{j=1}^{n} \mathbf{E}\left[\mathbf{H}^{\alpha} \mathbf{J R H}\right]_{j+n, j}, \\
t_{\alpha} & :=\frac{1}{n} \sum_{j=1}^{n} \mathbf{E}\left[\mathbf{H}^{\alpha} \mathbf{J R}\right]_{j j}, \quad u_{\alpha}:=\frac{1}{n} \sum_{j=1}^{n} \mathbf{E}\left[\mathbf{H}^{\alpha} \mathbf{J R}\right]_{j+n, j+n}
\end{aligned}
$$

We prove the following

Lemma 4.3. Assuming the conditions of Theorem 1.1 there exists constant $C>0$ such that the following inequality holds

$$
\begin{aligned}
\left|B_{2}+\sum_{q=0}^{m-1} f_{m-1, q} g_{m-1-q, 0}\right| & \leq \frac{C}{n v^{4}}, \\
\left|D_{2}+\sum_{q=0}^{m-1} g_{m-1, q} f_{m-1-q, 0}\right| & \leq \frac{C}{n v^{4}} .
\end{aligned}
$$

Proof. Consider the first inequality. Applying Hölder's inequality, we get

$$
\begin{aligned}
\mid B_{2}+ & \sum_{q=0}^{m-1} f_{m-1, q} g_{m-1-q, 0} \mid \\
\leq & \sum_{q=0}^{m-1} \mathbf{E}^{\frac{1}{2}}\left|\frac{1}{n} \sum_{j=1}^{n}\left(\left[\mathbf{H}^{m-1} \mathbf{J R} \mathbf{H}^{q}\right]_{j, j+n}-\mathbf{E} \sum_{j=1}^{n}\left[\mathbf{H}^{m-1} \mathbf{J R H}\right]_{j, j+n}\right)\right|^{2} \\
& \times \mathbf{E}^{\frac{1}{2}}\left|\frac{1}{n} \sum_{j=1}^{n}\left(\left[\mathbf{H}^{m-1-q} \mathbf{J R}\right]_{j+n, j}-\mathbf{E}\left[\mathbf{H}^{m-1-q} \mathbf{J R}\right]_{j+n, j}\right)\right|^{2}
\end{aligned}
$$

To conclude the proof of Lemma it is enough to use Lemmas 5.3 and 5.4 . The proof of the second inequality is similar. Thus the Lemma is proved. 
Note that

$$
f_{00}=g_{00}=\frac{1}{n} \sum_{j=1}^{n} \mathbf{E} \mathbf{R}_{j j}=\frac{1}{n} \sum_{j=1}^{n} \mathbf{E} \mathbf{R}_{j+n, j+n}=s_{n}(z) .
$$

By Lemma 4.3 and equality (4.13), we may write

$$
\begin{aligned}
B_{2}+D_{2}=-\frac{1}{2} s_{n}(z) & \left(f_{m-1, m-1}+g_{m-1, m-1}\right) \\
- & \frac{1}{2} \sum_{q=0}^{m-2}\left(f_{m-1, q} g_{m-1-q, 0}+g_{m-1, q} f_{m-1-q, 0}\right)+\varepsilon_{n}(z) .
\end{aligned}
$$

We consider now the behavior of the coefficients $f_{\alpha, \beta}, g_{\alpha, \beta}, t_{\alpha}$ and $u_{\alpha}$, for $\alpha, \beta=0, \ldots, m-$ 1. Applying Lemmas 5.7 and 5.4, we obtain the following relation for $\alpha>0, \beta>0$

$$
f_{\alpha, \beta}=-\sum_{q=0}^{m-1} f_{\alpha-1, q} t_{m-1+\beta-q}+f_{\alpha-1, \beta-1}+\varepsilon_{n}(z) .
$$

It is straightforward to check that for $q \geq m$ the following relation holds

$$
t_{q}=\frac{1}{n} \sum_{j=1}^{n}\left[\mathbf{H}^{q}\right]_{j+n, j+n}+z \frac{1}{n} \sum_{j=1}^{n}\left[\mathbf{H}^{q-m} \mathbf{R}\right]_{j+n, j+n}=\delta_{q}+z f_{q-m, 0}+\varepsilon_{n}(z),
$$

where $\delta_{0}=1$ and $\delta_{q}=0$ for $q>0$. Using relation (4.16), we may rewrite (4.15) in the following form

$$
f_{\alpha, \beta}=-z \sum_{q=0}^{\beta-1} f_{\alpha-1, q} f_{\beta-1-q, 0}-\sum_{q=\beta}^{m-1} f_{\alpha-1, q} u_{m-1+\beta-q}+\varepsilon_{n}(z) .
$$

For $\beta=0$, we get

$$
f_{\alpha, 0}=-\sum_{q=0}^{m-1} f_{\alpha-1, q} u_{m-1-q}+\varepsilon_{n}(z)
$$

Similar we obtain

$$
g_{\alpha, \beta}=-z \sum_{q=0}^{\beta-1} g_{\alpha-1, q} g_{\beta-1-q, 0}-\sum_{q=\alpha}^{m-1} g_{\alpha-1, q} t_{m-1+\beta-q}+\varepsilon_{n}(z) .
$$

and

$$
g_{\alpha, 0}=-\sum_{q=0}^{m-1} g_{\alpha-1, q} t_{m-1-q}+\varepsilon_{n}(z) .
$$

Applying Lemmas 5.7 and 5.4, we obtain a similar relation for $u_{\alpha}$ and $t_{\alpha}$, for $\alpha=0, \ldots, m-$ 1.

$$
u_{\alpha}=-f_{\alpha-1, m-1} g_{0,0}-\sum_{q=0}^{m-2} f_{\alpha-1, q} g_{m-1-q, 0}+\varepsilon_{n}(z),
$$


and

$$
t_{\alpha}=-g_{\alpha-1, m-1} f_{0,0}-\sum_{q=0}^{m-2} g_{\alpha-1, q} f_{m-1-q, 0}+\varepsilon_{n}(z) .
$$

Denote by $\mathbf{F}$ (resp. G) a $m-1 \times m-1$ matrix with entries $F_{p, q}=f_{p-1, q-1}$ (resp. $\left.\mathbf{G}_{p, q}=g_{p-1, q-1}\right), p, q=1 \ldots, m$. Let $\mathbf{t}$ (resp. u) denote a vector-column $\left(t_{1}, \ldots, t_{m-1}\right)^{T}$ $\left(\operatorname{resp}\left(u_{1}, \ldots, u_{m-1}\right)^{T}\right)$. Let $\mathbf{f}_{\alpha}=\left(f_{\alpha, 0}, \ldots, f_{\alpha, \alpha-1}, 0, f_{\alpha, \alpha+1}, \ldots, f_{\alpha, m-1}\right)^{T}$ and $\mathbf{g}_{\alpha}=$ $\left(g_{\alpha, 0}, \ldots, g_{\alpha, \alpha-1}, 0, g_{\alpha, \alpha+1}, \ldots, g_{\alpha, m-1}\right)^{T}$, for $\alpha=0, \ldots, m-2$. Introduce the matrices

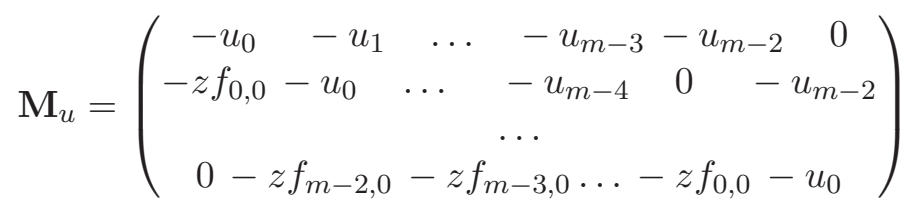

and

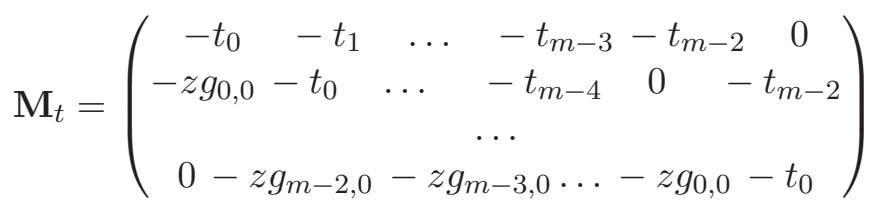

Let

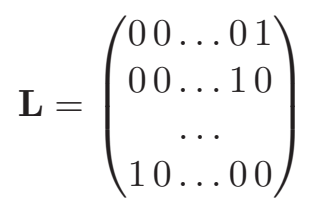

We introduce as well the vectors $\mathbf{y}_{\alpha}=\left(-f_{0, \alpha-1}, \ldots,-f_{0,1}, 0,-z f_{0,1}, \ldots,-z f_{m-\alpha, 0}\right)^{T}$ and $\mathbf{w}_{\alpha}=\left(-g_{0, \alpha-1}, \ldots,-g_{0,1}, 0,-z g_{0,1}, \ldots,-z g_{m-\alpha, 0}\right)^{T}$. We shall denote by $\mathbf{r}_{n}$ quantities such that $\left\|\mathbf{r}_{n}(z)\right\| \leq \frac{C \tau_{n}}{v^{4}}$.

Using these notations we may rewrite the relations (4.19)-4.22) as follows, for $\alpha=$ $1, \ldots, m$,

$$
\mathbf{g}_{\alpha}=g_{\alpha-1, \alpha-1} \mathbf{w}_{\alpha}+\mathbf{M}_{t} \mathbf{L} \mathbf{g}_{\alpha-1}+\mathbf{r}_{n}(z), \quad \mathbf{f}_{\alpha}=f_{\alpha-1, \alpha-1} \mathbf{y}_{\alpha}+\mathbf{M}_{u} \mathbf{L} \mathbf{f}_{\alpha-1}+\mathbf{r}_{n}(z),
$$

and

$$
\mathbf{t}=-s_{n}(z) \mathbf{f}_{m-1}+\mathbf{F L} \mathbf{f}_{0}+\mathbf{r}_{n}(z), \quad \mathbf{u}=-s_{n}(z) \mathbf{g}_{m-1}+\mathbf{G L g}_{0}+\mathbf{r}_{n}(z) .
$$

Furthermore, we may represent the relations (4.18) and (4.20) as follows

$$
\mathbf{f}_{0}=-u_{0} \mathbf{f}_{m-1}+\mathbf{F L u}+\mathbf{r}_{n}(z), \quad \mathbf{g}_{0}=-t_{0} \mathbf{g}_{m-1}+\mathbf{G L t}+\mathbf{r}_{n}(z) .
$$

Lemma 4.4. Under the conditions of Theorem 1.1 there exists a sufficiently large constant $V_{0}$ such that for any $v \geq V_{0}$ we have

$$
\max \left\{\|\mathbf{u}\|,\|\mathbf{t}\|,\left\|\mathbf{f}_{\alpha}\right\|,\left\|\mathbf{g}_{\alpha}\right\|\right\} \leq \frac{C \tau_{n}}{v^{4}} .
$$


Proof. First we note that, for $z=u+i v$ such that $v>0$

$$
\left\|\mathbf{w}_{\alpha}\right\|+\left\|\mathbf{y}_{\alpha}\right\| \leq C\left(\left\|\mathbf{f}_{0}\right\|+\left\|\mathbf{g}_{0}\right\|\right) .
$$

Furthermore, by Lemma 5.2 and inequality $\|\mathbf{R}\| \leq v^{-1}$, we have

$$
\max \{\|\mathbf{F}\|,\|\mathbf{G}\|\} \leq \frac{C_{m}}{v} .
$$

It is straightforward to check that

$$
\max \left\{\left|z f_{\alpha, \beta}\right|,\left|z g_{\alpha, \beta}\right|\right\} \leq C_{m}\left(1+\frac{1}{v}\right) .
$$

The last inequalities imply that

$$
\max \left\{\left\|\mathbf{M}_{u}\right\|,\left\|\mathbf{M}_{t}\right\|\right\} \leq C_{m}\left(1+\frac{1}{v}\right) .
$$

Relations (4.24), (4.25) together imply that

$$
\|\mathbf{u}\|+\|\mathbf{t}\| \leq \frac{C_{m}}{v}\left(\left\|\mathbf{f}_{m-1}\right\|+\left\|\mathbf{g}_{m-1}\right\|\right)+\left\|\mathbf{r}_{n}(z)\right\| .
$$

Relation (4.23) implies that

$$
\left\|\mathbf{g}_{m-1}\right\|+\left\|\mathbf{f}_{m-1}\right\| \leq \frac{C_{m}}{v} \sum_{q=1}^{m-1}\left(\left\|\mathbf{w}_{q}\right\|+\left\|\mathbf{y}_{q}\right\|\right)+C\left(\left\|\mathbf{g}_{0}\right\|+\left\|\mathbf{f}_{0}\right\|\right)+\left\|r_{n}(z)\right\| .
$$

Applying now inequality (4.26), we get

$$
\left\|\mathbf{g}_{m-1}\right\|+\left\|\mathbf{f}_{m-1}\right\| \leq C_{m}\left(\left\|\mathbf{f}_{0}\right\|+\left\|\mathbf{g}_{0}\right\|\right)+\left\|r_{n}(z)\right\| .
$$

Furthermore, relation (4.25) implies that

$$
\left\|\mathbf{f}_{0}\right\|+\left\|\mathbf{g}_{0}\right\| \leq \frac{C_{m}}{v}\left(\left\|\mathbf{g}_{m-1}\right\|+\left\|\mathbf{f}_{m-1}\right\|\right)+\frac{C}{v}(\|\mathbf{u}\|+\|\mathbf{t}\|)+\left\|r_{n}(z)\right\| .
$$

Inequalities (4.27), (4.28), (4.29) together imply

$$
\left\|\mathbf{g}_{m-1}\right\|+\left\|\mathbf{f}_{m-1}\right\| \leq \frac{C_{m}}{v}\left(\left\|\mathbf{g}_{m-1}\right\|+\left\|\mathbf{f}_{m-1}\right\|\right)+\left\|r_{n}(z)\right\| .
$$

Choosing $v_{0}$ such that $\frac{C_{m}}{v} \leq \frac{1}{4}$, we obtain

$$
\left\|\mathbf{g}_{m-1}\right\|+\left\|\mathbf{f}_{m-1}\right\| \leq \frac{C_{m} \tau_{n}}{v^{4}} .
$$

Relation (4.27) implies now that

$$
\|\mathbf{u}\|+\|\mathbf{t}\| \leq \frac{C_{m} \tau_{n}}{v^{4}}
$$


From relation (4.26) it follows that

$$
\left\|\mathbf{w}_{\alpha}\right\|+\left\|\mathbf{y}_{\alpha}\right\| \leq \frac{C \tau_{n}}{v^{4}}
$$

Similar to inequality (4.31) we get

$$
\left\|\mathbf{g}_{\alpha}\right\|+\left\|\mathbf{f}_{\alpha}\right\| \leq \frac{C \tau_{n}}{v^{4}}
$$

Thus the Lemma is proved.

Lemma 4.5. Under the conditions of Theorem 1.1 we have

$$
f_{\alpha, \alpha}=-z s(z) f_{\alpha-1, \alpha-1}+\varepsilon_{n}(z) .
$$

and

$$
g_{\alpha, \alpha}=-z s(z) g_{\alpha-1, \alpha-1}+\varepsilon_{n}(z) .
$$

Proof. We shall consider the first equality only, the proof of the other one being similar. By relation (4.17), we have

$$
f_{\alpha, \alpha}=-z f_{0,0} f_{\alpha-1, \alpha-1}-z \sum_{q=0}^{\alpha-2} f_{\alpha-1, q} f_{\alpha-1-q, 0}-\sum_{q=\alpha}^{m-1} f_{\alpha-1, q} u_{m-1+\beta-q}+\varepsilon_{n}(z) .
$$

This equality implies that

$$
f_{\alpha, \alpha}=-z s(z) f_{\alpha-1, \alpha-1}+\theta|z|\left\|\mathbf{f}_{\alpha-1}\right\|\left\|\mathbf{f}_{0}\right\|+\varepsilon_{n}(z) .
$$

Applying Lemma 4.4 we conclude the proof.

Equality (4.14) and Lemma 4.5 together imply

$$
1+z s_{n}(z)=-s(z)\left(f_{m-1, m-1}+g_{m-1, m-1}\right)+\varepsilon_{n}(z)=(-1)^{m} z^{m-1} s_{n}^{m+1}(z)+\varepsilon_{n}(z)
$$

We rewrite that last equation as follows

$$
1+z s_{n}(z)+(-1)^{m-1} z^{m-1} s_{n}^{m+1}(z)=\varepsilon_{n}(z)
$$

The Stieltjes transform $s(z)$ satisfies the equation

$$
1+z s(z)+(-1)^{m-1} z^{m-1} s^{m+1}(z)=0
$$

The last two relations together imply that, for $v \geq V_{0}$

$$
\left|s_{n}(z)-s(z)\right| \leq \frac{\left|\varepsilon_{n}(z)\right|}{\left|z+(-z)^{m-1} \sum_{q=0}^{m} s^{q}(z) s_{n}^{m-q}(z)\right|}
$$


Note that

$$
\max \left\{|z s(z)|,\left|z s_{n}(z)\right|\right\} \leq C\left(1+\frac{1}{v}\right)
$$

and

$$
\max \left\{\left|s_{n}(z)\right|,|s(z)|\right\} \leq \frac{1}{v}
$$

Applying these inequality, we obtain

$$
\left|(-z)^{m-1} \sum_{q=0}^{m} s^{q}(z) s_{n}^{m-q}(z)\right| \leq \frac{C}{v} .
$$

We may choose $V_{1} \geq V_{0}$ such that for any $v \geq V_{1}$

$$
\frac{C}{v} \leq \frac{v}{2}
$$

This implies that for $v \geq V_{1}$

$$
\left|\operatorname{Im}\left\{z+(-z)^{m-1} \sum_{q=0}^{m} s^{q}(z) s_{n}^{m-q}(z)\right\}\right| \geq \frac{v}{2}
$$

and

$$
\left|s_{n}(z)-s(z)\right| \leq \frac{C \tau_{n}}{v^{4}} .
$$

From inequality (4.45) we conclude that there exists an open set with non-empty interior such that $s_{n}(z)$ converges to $s(z)$ on this set. The Stieltjes transform of these random variables is an analytic function on $\mathcal{C}^{+}$and locally bounded $\left(\left|s_{n}(z)\right| \leq v^{-1}\right.$ for any $\left.v>0\right)$. By Montel's Theorem (see, for instance, 4, p. 153, Theorem 2.9) the convergence of $s_{n}(z)$ to $s(z)$ is uniform on any compact set in the upper half-plane $\mathcal{K} \subset \mathcal{C}^{+}$. This implies that $\Delta_{n} \rightarrow 0$ as $n \rightarrow \infty$. Thus the proof of Theorem 1.1 in the general case is complete.

\section{Appendix}

\subsection{Frobenius norms of powers of truncated matrices}

Recall that we consider truncated independent random variable $X_{j k}$ satisfying

$$
\left|\mathbf{E} X_{j k}\right| \leq \frac{1}{n^{\frac{3}{2}} \tau_{n}}, \quad \mathbf{E}\left|X_{j k}\right|^{2}=1+\frac{\theta_{j k} M}{n^{2} \tau_{n}^{2}}, \quad\left|X_{j k}\right| \leq \tau_{n} \sqrt{n},
$$

where $\tau_{n} \rightarrow 0$ as $n \rightarrow \infty$ converges to zero as slowly as needed.

We would like to investigate the behavior of the Frobenius norm of powers $\left\|\mathbf{X}^{m}\right\|_{2}$ of the random matrix $\mathbf{X}=\frac{1}{\sqrt{n}}\left(X_{j k}\right)_{j, k=1}^{n}$. We formulate the following 
Lemma 5.1. Let $X_{j k}^{(n)}$ be independent random variables for $1 \leq j, k \leq n$ and assume that (5.1) holds. Then for any $m \geq 1$ and any $\nu=0, \ldots, m$, there exists a constant $C_{m}>0$ depending on $m$ such that

$$
\mathbf{E}\left\|\mathbf{X}^{\nu}(\mathbf{X}-\mathbf{E} \mathbf{X})^{m-\nu}\right\|_{2}^{2} \leq C_{m} n
$$

Proof. We start with the case $\nu=0$. Consider the matrix $\widetilde{\mathbf{X}}:=\mathbf{X}-\mathbf{E} \mathbf{X}=\frac{1}{\sqrt{n}}\left(\widetilde{X}_{j k}\right)_{j, k=1}^{n}$ and the norms of the powers of this matrix. We may write

$$
\begin{array}{r}
\mathbf{E}\|\widetilde{\mathbf{X}}\|_{2}^{2}=n^{-m} \sum_{j, k=1}^{n} \sum_{j_{1}, \ldots, j_{m-1}=1}^{n} \sum_{j_{1}^{\prime}, \ldots, j_{m-1}^{\prime}=1}^{n} \mathbf{E} \widetilde{X}_{j_{1}} \tilde{X}_{j_{1} j_{2}} \ldots \widetilde{X}_{j_{m-2} j_{m-1}} \widetilde{X}_{j_{m-1} k} \\
\overline{\widetilde{X}}_{j j_{1}^{\prime}} \overline{\widetilde{X}}_{j_{1}^{\prime} j_{2}^{\prime}} \ldots \overline{\widetilde{X}}_{j_{m-2}^{\prime} j_{m-1}^{\prime}} \overline{\widetilde{X}}_{j_{m-1}^{\prime} k} .
\end{array}
$$

Here for any number $a=u+\sqrt{-1} v, \bar{a}=u-\sqrt{-1} v$ denotes the complex conjugate. The product in the right hand side of (5.3) involves $\mu$ different (with respect to complex conjugates) terms, say $X_{l_{1}, l_{1}^{\prime}}^{\varepsilon}, \ldots, X_{l_{\mu}, l_{\mu}^{\prime}}^{\varepsilon}$, with multiplicities $m_{1}, \ldots, m_{\mu}$, where $\varepsilon= \pm$ and

$$
X_{j k}^{\varepsilon}=\left\{\begin{array}{lll}
X_{j k}, & \text { if } & \varepsilon=+ \\
\bar{X}_{j k}, & \text { if } & \varepsilon=-
\end{array}\right.
$$

Note that $m_{1}+\cdots+m_{\mu}=2 m$ and if $\min \left\{m_{1}, \ldots, m_{\mu}\right\}=1$ then expectation of corresponding product equals 0 since $\mathbf{E} X_{j, l}^{\varepsilon}=0$ for any $j, l=1, \ldots, n$. This implies that non zero terms occur for $\mu \leq m$ and $\min \left\{m_{1}, \ldots, m_{\mu}\right\} \geq 2$ only. By assumption (5.1), we have

$$
\left|\mathbf{E} \widetilde{X}_{j j_{1}} \tilde{X}_{j_{1} j_{2}} \cdots \widetilde{X}_{j_{m-2} j_{m-1}} \widetilde{X}_{j_{m-1} k} \overline{\widetilde{X}}_{j j_{1}^{\prime}} \overline{\widetilde{X}}_{j_{1}^{\prime} j_{2}^{\prime}} \cdots \overline{\widetilde{X}}_{j_{m-2}^{\prime} j_{m-1}^{\prime}} \overline{\widetilde{X}}_{j_{m-1}^{\prime} k}\right| \leq n^{m-\mu} \tau_{n}^{2(m-\mu)} .
$$

The cardinality $\mathcal{N}\left(l_{1}, \ldots, l_{m}, l_{1}^{\prime}, \ldots, l_{m}^{\prime}\right)$ of the set of indices with $\mu$ different edges $l_{\nu}, l_{\nu}^{\prime}$ and multiplicities $m_{1}, \ldots, m_{\mu}$ respectively satisfies the inequality

$$
\mathcal{N}\left(l_{1}, \ldots, l_{m}, l_{1}^{\prime}, \ldots, l_{m}^{\prime}\right) \leq C n^{\mu+1} .
$$

The representation (5.3) and the inequalities (5.5) and (5.6) together imply

$$
\mathbf{E}\left\|\widetilde{\mathbf{X}}^{m}\right\|_{2}^{2} \leq C_{m} n
$$

Assume now that $1 \leq \nu \leq m$. Consider the quantity

$$
\Gamma_{n}^{(\nu)}=\mathbf{E}\left\|\mathbf{X}^{\nu}(\mathbf{X}-\mathbf{E} \mathbf{X})^{m-\nu}\right\|_{2}^{2} .
$$

Let $\mathbf{A}=\mathbf{E} \mathbf{X}$. It is straightforward to check that

$$
\Gamma_{n}^{(\nu)} \leq C_{m} \sum_{\alpha=0}^{\nu}\left(\mathbf{E}\|\mathbf{A}\|_{2}^{2}\right)^{\alpha} \mathbf{E}\left\|(\mathbf{X}-\mathbf{A})^{m-\alpha}\right\|_{2}^{2} .
$$


To prove (5.9) we consider the representation

$$
\mathbf{X}^{\nu}=\sum^{*} \mathbf{A}^{m_{1}}(\mathbf{X}-\mathbf{A})^{m_{1}^{\prime}} \cdots \mathbf{A}^{m_{\nu}}(\mathbf{X}-\mathbf{A})^{m_{\nu}^{\prime}}
$$

where $\sum^{*}$ stands for sum over all indices $m_{1}, \ldots, m_{k}, m_{1}^{\prime}, \ldots, m_{\nu}^{\prime} \geq 0$ such that $m_{1}+\cdots+m_{\nu}+m_{1}^{\prime}+\cdots+m_{\nu}^{\prime}=\nu$. This implies the bound

$$
\Gamma_{n}^{(\nu)} \leq C_{m} \sum^{*} \mathbf{E}\left\|\mathbf{A}^{m_{1}}(\mathbf{X}-\mathbf{A})^{m_{1}^{\prime}} \cdots \mathbf{A}^{m_{\nu}}(\mathbf{X}-\mathbf{A})^{m_{\nu}^{\prime}}\right\|_{2}^{2}
$$

Using that for any matrices $\mathbf{A}$ and $\mathbf{B}$ we have $\|\mathbf{A B}\|_{2}=\|\mathbf{B A}\|_{2}$ and that $\left\|\mathbf{A}^{\nu}\right\|_{2} \leq\|\mathbf{A}\|_{2}^{\nu}$, we get from this inequality the bound (5.9). By assumption (5.1), we have

$$
\|\mathbf{A}\|_{2}^{2} \leq \frac{C M}{n^{2} \tau_{n}^{6}} .
$$

Inequalities (5.11), (5.12), (5.7) and the induction assumption together conclude the proof of the Lemma.

We shall use the following obvious bounds

Lemma 5.2. Let $X_{j k}^{(n)}$ be independent random variables for $1 \leq j, k \leq n$. assume that (5.1) holds and that $\mathbf{E} X_{j k}=0$. Then for any $m, r \geq 1$ and any $\nu=0, \ldots, m$, and any $j=1, \ldots, n$, there exists a constant $C(m, r)>0$ depending on $m, r$ such that

$$
\max \left\{\mathbf{E}\left\|\mathbf{X}^{\nu} \mathbf{e}_{j}\right\|_{2}^{2 r}, \mathbf{E}\left\|\mathbf{e}_{j}^{T} \mathbf{X}^{\nu}\right\|_{2}^{2 r}\right\} \leq C(m, r) .
$$

Proof. Let

$$
\Gamma_{\nu, j}=\left\|\mathbf{X}^{\nu} \mathbf{e}_{j}\right\|_{2}
$$

We may write

$$
\begin{aligned}
\Gamma_{\nu, j}^{2}=\sum_{k_{1}, \ldots, k_{r}=1}^{n} \sum_{j_{1}^{(1)}, \ldots, j_{\nu-1}^{(1)}=1}^{n} \sum_{j_{1}^{(1)^{\prime}}, \ldots, j_{\nu-1}^{(1)}{ }^{\prime}=1}^{n} \ldots \sum_{j_{1}^{(r)}, \ldots, j_{\nu-1}^{(r)}=1}^{n} & \\
& \times \sum_{j_{1}^{(r)^{\prime}, \ldots, j_{\nu-1}^{(r)}{ }^{\prime}=1}}^{n} \prod_{q=1}^{r} A\left(k_{q}, j_{1}^{(q)}, \ldots, j_{\nu-1}^{(q)}\right) \bar{A}\left(k_{q}, j_{1}^{(q)^{\prime}}, \ldots, j_{\nu-1}^{(q)}{ }^{\prime}\right),
\end{aligned}
$$

where

$$
A\left(k_{q}, j_{1}^{(q)}, \ldots, j_{\nu-1}^{(q)}\right)=X_{k_{q} j_{1}^{(q)}} X_{j_{1}^{(q)} j_{2}^{(1)}} \cdots X_{j_{\nu-2}^{(q)} j_{\nu-1}^{(q)}} X_{j_{\nu-1}^{(q)}} .
$$

Assume that the set of indices $\mathcal{N}=\cup_{q=1}^{r}\left\{\left\{k_{q}, j_{1}^{(q)}, \ldots, j_{\nu-1}^{(q)}\right\} \cup j_{1}^{(q)^{\prime}}, \ldots, j_{\nu-1}^{(q)}{ }^{\prime}\right\}$ consists of $\mu$ different pairs, say $l_{1}, l_{1}^{\prime}, \ldots, l_{\mu}, l_{\mu}^{\prime}$, with multiplicities $m_{1}, \ldots, m_{\mu}$ respectively. Note that $m_{1}+\cdots+m_{\mu}=2 q r$ and if $\min \left\{m_{1}, \ldots, m_{\mu}\right\}=1$ then the corresponding term equals 0 , 
since $\mathbf{E} X_{j, l}=0$ for any $j, l=1, \ldots, n$. This implies that $\mu \leq m$ and $\min \left\{m_{1}, \ldots, m_{\mu}\right\} \geq 2$. By assumption (5.1), we have

$$
\mid \mathbf{E} A\left(k_{q}, j_{1}^{(q)}, \ldots, j_{\nu-1}^{(q)}\right) \bar{A}\left(k_{q}, j_{1}^{(q)^{\prime}}, \ldots, j_{\nu-1}^{(q)}{ }^{\prime} \mid \leq C\left(\tau_{n} \sqrt{n}\right)^{2 m r-2 \mu} .\right.
$$

The cardinality $\mathcal{N}\left(l_{1}, \ldots, l_{m}, l_{1}^{\prime}, \ldots, l_{m}^{\prime}\right)$ of the set of indices with $\mu$ different edges $l_{\nu}, l_{\nu}$ and multiplicities $m_{1}, \ldots, m_{\mu}$ satisfies the inequality

$$
\mathcal{N}\left(l_{1}, \ldots, l_{m}, l_{1}^{\prime}, \ldots, l_{m}^{\prime}\right) \leq C n^{\mu} .
$$

The representation (5.3) and the inequalities (5.17) and (5.18) together imply

$$
\mathbf{E}\left\|\mathbf{X}^{\nu} \mathbf{e}_{j}\right\|_{2}^{2 r} \leq C(m, r)
$$

The bound of $\mathbf{E}\left\|\mathbf{e}_{j}^{T} \mathbf{X}^{\nu}\right\|_{2}^{2 r}$ is similar. Thus, lemma is proved.

Lemma 5.3. Under the conditions of Theorem 1.1 we have

$$
\mathbf{E}\left|\frac{1}{n}(\operatorname{Tr} \mathbf{R}-\mathbf{E} \operatorname{Tr} \mathbf{R})\right|^{2} \leq \frac{C}{n v^{2}} .
$$

Proof. Consider the matrix $\mathbf{X}^{(j)}$ obtained from the matrix $\mathbf{X}$ by replacing the entries of the $j$-th row by zeros. We define the following matrices

$$
\mathbf{H}^{(j)}=\left(\begin{array}{cc}
\mathbf{X}^{(j)} & \mathbf{O} \\
\mathbf{O} & \mathbf{X}^{(j)^{*}}
\end{array}\right), \quad \widehat{\mathbf{H}}^{(j)}=\mathbf{H}^{(j)} \mathbf{J} .
$$

We shall use the following inequality. For any Hermitian matrix $\mathbf{A}$ and $\mathbf{B}$ with spectral distribution function $F_{A}(x)$ and $F_{B}(x)$ respectively, we have

$$
\left|\operatorname{Tr}(\mathbf{A}-z \mathbf{I})^{-1}-\operatorname{Tr}(\mathbf{B}-z \mathbf{I})^{-1}\right| \leq \frac{\operatorname{rank}(\mathbf{A}-\mathbf{B})}{v} .
$$

It is straightforward to show that

$$
\operatorname{rank}\left(\mathbf{H}^{q} \mathbf{J}-\mathbf{H}^{(j)^{q}} \mathbf{J}\right) \leq 4 q .
$$

Inequalities (5.22) and (5.23) together imply

$$
\left|\frac{1}{2 n}\left(\operatorname{Tr} \mathbf{R}-\operatorname{Tr} \mathbf{R}^{(j)}\right)\right| \leq \frac{C}{n v} .
$$

After this remark we may apply a well-known martingale expansion techniques suggested already by Girko [5]. We may introduce $\sigma$-algebras $\mathcal{F}_{j}=\sigma\left\{X_{l k}, j<l \leq n, k=1, \ldots, n\right\}$ and use the representation

$$
\operatorname{Tr} \mathbf{R}-\mathbf{E} \operatorname{Tr} \mathbf{R}=\sum_{\nu=1}^{m} \sum_{j=1}^{n}\left(\mathbf{E}_{j-1} \operatorname{Tr} \mathbf{R}-\mathbf{E}_{j} \operatorname{Tr} \mathbf{R}\right),
$$

where $\mathbf{E}_{j}$ denotes conditional expectation given $\sigma$-algebra $\mathcal{F}_{j}$. 
Lemma 5.4. Under the conditions of Theorem 1.1 we have, for $q \geq 1$

$$
\mathbf{E}\left|\frac{1}{n}\left(\sum_{j=1}^{n}\left[\mathbf{H}^{q} \mathbf{J R}\right]_{j j+n}-\mathbf{E} \sum_{j=1}^{n}\left[\mathbf{H}^{q} \mathbf{J R}\right]_{j j+n}\right)\right|^{2} \leq \frac{C}{n v^{4}} .
$$

Proof. We introduce the matrices $\mathbf{X}^{(j)}=\mathbf{X}-\mathbf{e}_{j} \mathbf{e}_{j}^{T} \mathbf{X}$, and $\mathbf{H}^{(j)}=\mathbf{H}-\mathbf{e}_{j} \mathbf{e}_{j}^{T} \mathbf{H}-\mathbf{H} \mathbf{e}_{j+n} \mathbf{e}_{j+n}^{T}$. Note that the matrix $\mathbf{X}^{(j)}$ is obtained from the matrix $\mathbf{X}$ by replacing the entries of the $j$-th row by 0 . Consider the quantity

$$
S_{j}:=\sum_{k=1}^{n}\left[\mathbf{H}^{q} \mathbf{J R}\right]_{k k+n}-\sum_{k=1}^{n}\left[\mathbf{H}^{(j)^{q}} \mathbf{J R}^{(j)}\right]_{k k+n} .
$$

Using equality

$$
\begin{aligned}
\mathbf{H}^{q} \mathbf{J R}-\mathbf{H}^{(j)^{q}} \mathbf{J R}^{(j)} & =\sum_{\nu=0}^{q-1} \mathbf{H}^{(j)^{\nu}}\left(\mathbf{H}-\mathbf{H}^{(j)}\right) \mathbf{H}^{q-1-\nu} \mathbf{J R} \\
& +\sum_{\nu=0}^{p-1} \mathbf{H}^{(j)^{q}} \mathbf{J} \mathbf{R}^{(j)} \mathbf{H}^{\nu}\left(\mathbf{H}-\mathbf{H}^{(j)}\right) \mathbf{H}^{p-1-\nu} \mathbf{J R}
\end{aligned}
$$

we get

$$
S_{j}=S_{j}^{(1)}+S_{j}^{(2)}
$$

where

$$
\begin{gathered}
S_{j}^{(1)}=\sum_{\nu=0}^{q-1} \sum_{k=1}^{n}\left[\mathbf{H}^{(j)^{\nu}}\left(\mathbf{H}-\mathbf{H}^{(j)}\right) \mathbf{H}^{q-1-\nu} \mathbf{J R}\right]_{k k+n} \\
S_{j}^{(2)}=\sum_{\nu=0}^{p-1} \sum_{k=1}^{n}\left[\mathbf{H}^{(j)^{q}} \mathbf{J} \mathbf{R}^{(j)} \mathbf{H}^{\nu}\left(\mathbf{H}-\mathbf{H}^{(j)}\right) \mathbf{H}^{p-1-\nu} \mathbf{J R}\right]_{k k+n} .
\end{gathered}
$$

Applying now that

$$
\mathbf{H}-\mathbf{H}^{(j)}=\mathbf{e}_{j} \mathbf{e}_{j}^{T} \mathbf{H}+\mathbf{H e}_{j+n} \mathbf{e}_{j+n}^{T},
$$

we obtain

$$
\begin{array}{r}
\sum_{k=1}^{n}\left[\mathbf{H}^{(j)^{\nu}}\left(\mathbf{H}-\mathbf{H}^{(j)}\right) \mathbf{H}^{q-1-\nu} \mathbf{J R}\right]_{k k+n}=\operatorname{Tr} \widehat{\mathbf{J}} \mathbf{H}^{(j)^{\nu}}\left(\mathbf{e}_{j} \mathbf{e}_{j}^{T} \mathbf{H}+\mathbf{H e}_{j+n} \mathbf{e}_{j+n}^{T}\right) \mathbf{H}^{q-1-\nu} \mathbf{J R} \\
=\left[\mathbf{H}^{q-\nu} \mathbf{J R J H} \mathbf{H}^{(j)^{\nu}}\right]_{j j}+\left[\mathbf{H}^{q-1-\nu} \mathbf{J} \mathbf{R J} \mathbf{H}^{(j)^{\nu}} \mathbf{H}\right]_{j+n j+n} \mid .
\end{array}
$$

Here

$$
\widehat{J}=\left(\begin{array}{cc}
\mathbf{O} & \mathbf{I} \\
\mathbf{O} & \mathbf{O}
\end{array}\right)
$$


Equality (5.31) implies that

$$
\left|S_{j}^{(1)}\right| \leq \sum_{\nu=0}^{q-1}\left|\left[\mathbf{H}^{q-\nu} \mathbf{J R J} \mathbf{H}^{(j)^{\nu}}\right]_{j j}\right|+\left|\left[\mathbf{H}^{q-1-\nu} \mathbf{J} \mathbf{R} \mathbf{J H}^{(j)^{\nu}} \mathbf{H}\right]_{j+n j+n}\right| .
$$

Using Hölder's inequality, we get

$$
\mathbf{E}\left|S_{j}^{(1)}\right|^{2} \leq \frac{C}{v^{2}} \sum_{\nu=0}^{q-1}\left(\mathbf{E}\left\|\mathbf{e}_{j}^{T} \mathbf{H}^{q-\nu}\right\|_{2}^{2}\left\|\mathbf{H}^{(j)^{\nu}} \mathbf{e}_{j}\right\|_{2}^{2}+\mathbf{E}\left\|\mathbf{e}_{j+n} \mathbf{H}^{q-1-\nu}\right\|_{2}^{2}\left\|\mathbf{H}^{(j)^{\nu}} \mathbf{H e}_{j+n}\right\|_{2}^{2}\right)
$$

Lemma 5.2 and Hölder's inequality together imply

$$
\mathbf{E}\left|S_{j}^{(1)}\right| \leq \frac{C}{v^{2}}
$$

Similar we get

$$
\mathbf{E}\left|S_{j}^{(2)}\right|^{2} \leq \frac{C}{v^{4}}
$$

Inequalities (5.35) and (5.36) together imply

$$
\mathbf{E}\left|S_{j}\right|^{2} \leq \frac{C\left(v^{2}+1\right)}{v^{4}} .
$$

Let $\mathcal{F}_{j}$ denote the $\sigma$-algebra generated by $X_{l k}$, for $1 \leq l \leq j, 1 \leq k \leq n$. Denote by $\mathbf{E}_{j}$ the conditional expectation with respect to $\sigma$-algebra $\mathcal{F}_{j}$. We may write

$$
\begin{aligned}
\mathbf{E}\left|\frac{1}{n}\left(\sum_{j=1}^{n}\left[\mathbf{H}^{q} \mathbf{J R}\right]_{j j+n}-\mathbf{E} \sum_{j=1}^{n}\left[\mathbf{H}^{q} \mathbf{J R}\right]_{j j+n}\right)\right|^{2} \\
\left.\quad=\frac{1}{n^{2}} \sum_{j=1}^{n} \mathbf{E} \mid \mathbf{E} \sum_{k=1}^{n}\left[\mathbf{H}^{q} \mathbf{J R}\right]_{k k+n}-\mathbf{E}_{j-1} \sum_{k=1}^{n}\left[\mathbf{H}^{q} \mathbf{J R}\right]_{k k+n}\right)\left.\right|^{2} \\
\left.\leq \frac{1}{n^{2}} \sum_{j=1}^{n} \mathbf{E} \mid \sum_{k=1}^{n}\left[\mathbf{H}^{q} \mathbf{J R}\right]_{k k+n}-\sum_{k=1}^{n}\left[\mathbf{H}^{(j)^{q}} \mathbf{J R}^{(j)}\right]_{k k+n}\right)\left.\right|^{2} \\
\leq \frac{C\left(1+v^{2}\right)}{n v^{4}} .
\end{aligned}
$$

Thus the Lemma is proved.

Lemma 5.5. Under the conditions of Theorem 1.1 the following inequality holds

$$
\mathbf{E}\left|\frac{1}{n}\left(\sum_{j=1}^{n} \mathbf{R}_{j, j+n}-\mathbf{E} \sum_{j=1}^{n} \mathbf{R}_{j, j+n}\right)\right|^{2} \leq \frac{C}{n v^{4}} .
$$


Proof. The proof is similar to the proof of the previous lemma. We have

$$
\sum_{k=1}^{n} \mathbf{R}_{k k+n}-\sum_{k=1}^{n} \mathbf{R}_{k k+n}^{(j)}=\sum_{\nu=0}^{p-1} \sum_{k=1}^{n}\left[\mathbf{R}^{(j)} \mathbf{H}^{(j)^{\nu}}\left(\mathbf{e}_{j} \mathbf{e}_{j}^{T} \mathbf{H}+\mathbf{H} \mathbf{e}_{j+n} \mathbf{e}_{j+n}^{T}\right) \mathbf{H}^{p-1-\nu} \mathbf{R}\right]_{k k+n} .
$$

Applying Hölder's inequality and inequality $\max \left\{\|\mathbf{R}\|,\left\|\mathbf{R}^{(j)}\right\|\right\} \leq v^{-1}$, we get

$$
\begin{aligned}
\left|\sum_{k=1}^{n} \mathbf{R}_{k k+n}-\sum_{k=1}^{n} \mathbf{R}_{k k+n}^{(j)}\right| & \leq \frac{1}{v^{2}} \sum_{\nu=0}^{p-1}\left\|\mathbf{H}^{(j)^{\nu}} \mathbf{e}_{j}\right\|_{2}\left\|\mathbf{e}_{j}^{T} \mathbf{H}^{p-\nu}\right\|_{2} \\
& +\frac{1}{v^{2}} \sum_{\nu=0}^{p-1}\left\|\mathbf{e}_{j+n}^{T} \mathbf{H}^{p-1-\nu}\right\|_{2}\left\|\mathbf{H}^{(j)^{\nu}} \mathbf{H} \mathbf{e}_{j+n}\right\|_{2} .
\end{aligned}
$$

Using Hölder inequality and Lemma 5.2 , we get

$$
\mathbf{E}\left|\sum_{k=1}^{n} \mathbf{R}_{k k+n}-\sum_{k=1}^{n} \mathbf{R}_{k k+n}^{(j)}\right|^{2} \leq \frac{C_{m}}{v^{4}} .
$$

To conclude the proof it is enough to use the martingale expansion of the difference $\sum_{k=1}^{n} R_{k k+n}-\sum_{k=1}^{n} \mathbf{E} R_{k k+n}$ similar to previous lemma.

Lemma 5.6. Under the conditions of Theorem 1.1 we have, for $0 \leq \mu, \nu \leq m$, that there exists a constant $C_{m}$ depending on $m$ such that

$$
\left|n^{-\frac{3}{2}} \sum_{j, k=1}^{n} \mathbf{E}\left(X_{j k}+X_{j k}^{3}\right)\left[\frac{\partial^{2}\left(\mathbf{H}^{\nu} \mathbf{J} \mathbf{R} \mathbf{H}^{\mu}\right)}{\partial X_{j k}^{2}}\left(\theta_{j k} X_{j k}\right)\right]_{k j}\right| \leq \frac{C_{m}(1+v)}{\sqrt{n} v^{3}},
$$

where $\theta_{j k}$ and $X_{j k}$ are mutually independent $j, k=1, \ldots, n$, and $\theta_{j k}$ are uniformly distributed on the unit interval. By $\frac{\partial^{2}}{\partial X_{j k}{ }^{2}} \mathbf{A}\left(\theta_{j k} X_{j k}\right)$ we denote the matrix obtained from $\frac{\partial^{2}}{\partial X_{j k}{ }^{2}} \mathbf{A}$ by replacing the entries $X_{j k}$ by $\theta_{j k} X_{j k}$.

Proof. By the formula for derivatives of a resolvent matrix, we have

$$
\begin{aligned}
\frac{\partial\left(\mathbf{H}^{\nu} \mathbf{J} \mathbf{R H}\right.}{\left.\partial X_{j k}\right)} & =\frac{1}{\sqrt{n}} \sum_{a=0}^{\nu-1} \mathbf{H}^{a}\left(\mathbf{e}_{j} \mathbf{e}_{k}^{T}+\mathbf{e}_{k+n} \mathbf{e}_{j+n}^{T}\right) \mathbf{H}^{\nu-1-a} \mathbf{J} \mathbf{R} \mathbf{H}^{\mu} \\
& -\frac{1}{\sqrt{n}} \sum_{b=0}^{m-1} \mathbf{H}^{\nu} \mathbf{J} \mathbf{R} \mathbf{H}^{b}\left(\mathbf{e}_{j} \mathbf{e}_{k}^{T}+\mathbf{e}_{k+n} \mathbf{e}_{j+n}^{T}\right) \mathbf{H}^{m-1-b} \mathbf{J} \mathbf{R} \mathbf{H}^{(\mu)} \\
& +\sum_{c=0}^{\mu-1} \mathbf{H}^{\nu} \mathbf{J R H}^{c}\left(\mathbf{e}_{j} \mathbf{e}_{k}^{T}+\mathbf{e}_{k+n} \mathbf{e}_{j+n}^{T}\right) \mathbf{H}^{\mu-1-c} .
\end{aligned}
$$


From this formula it follows that

$$
\frac{\partial^{2}\left(\mathbf{H}^{\nu} \mathbf{J} \mathbf{R H} \mathbf{H}^{\mu}\right)}{\partial X_{j k}^{2}}=\frac{1}{n}\left(\sum_{a=0}^{\nu-1} \sum_{i=1}^{4} \mathbf{P}_{i}^{(a)}+\sum_{c=1}^{\mu-1} \sum_{i=1}^{4} \mathbf{T}_{i}^{(c)}\right)-\frac{1}{n} \sum_{b=1}^{m-1} \sum_{i=1}^{6} \mathbf{U}_{i}^{(b)},
$$

where

$$
\begin{aligned}
& \mathbf{P}_{1}^{(a)}=-\sum_{s=0}^{a-1} \mathbf{H}^{s}\left(\mathbf{e}_{j} \mathbf{e}_{k}^{T}+\mathbf{e}_{k+n} \mathbf{e}_{j+n}^{T}\right) \mathbf{H}^{a-1-s}\left(\mathbf{e}_{j} \mathbf{e}_{k}^{T}+\mathbf{e}_{k+n} \mathbf{e}_{j+n}^{T}\right) \mathbf{H}^{\nu-1-a} \mathbf{J R} \mathbf{H}^{\mu} \\
& \mathbf{P}_{2}^{(a)}=-\frac{1}{n} \sum_{s=0}^{\nu-a-1} \mathbf{H}^{a}\left(\mathbf{e}_{j} \mathbf{e}_{k}^{T}+\mathbf{e}_{k+n} \mathbf{e}_{j+n}^{T}\right) \mathbf{H}^{s}\left(\mathbf{e}_{j} \mathbf{e}_{k}^{T}+\mathbf{e}_{k+n} \mathbf{e}_{j+n}^{T}\right) \mathbf{H}^{\nu-1-a-s} \mathbf{J} \mathbf{R} \mathbf{H}^{\mu} \\
& \mathbf{P}_{3}^{(a)}=-\frac{1}{n} \sum_{s=0}^{m-1} \mathbf{H}^{a}\left(\mathbf{e}_{j} \mathbf{e}_{k}^{T}+\mathbf{e}_{k+n} \mathbf{e}_{j+n}^{T}\right) \mathbf{H}^{\nu-1-s} \mathbf{J} \mathbf{R} \mathbf{H}^{s}\left(\mathbf{e}_{j} \mathbf{e}_{k}^{T}+\mathbf{e}_{k+n} \mathbf{e}_{j+n}^{T}\right) \mathbf{H}^{m-1-s} \mathbf{J R} \mathbf{H}^{\mu} \\
& \mathbf{P}_{4}^{(a)}=-\frac{1}{n} \sum_{s=0}^{\mu-1} \mathbf{H}^{a}\left(\mathbf{e}_{j} \mathbf{e}_{k}^{T}+\mathbf{e}_{k+n} \mathbf{e}_{j+n}^{T}\right) \mathbf{H}^{\nu-1-s} \mathbf{J R H}^{s}\left(\mathbf{e}_{j} \mathbf{e}_{k}^{T}+\mathbf{e}_{k+n} \mathbf{e}_{j+n}^{T}\right) \mathbf{H}^{\mu-1-s} .
\end{aligned}
$$

Furthermore,

$$
\begin{aligned}
& \mathbf{T}_{1}^{(c)}=-\frac{1}{n} \sum_{s=0}^{\nu-1} \mathbf{H}^{s}\left(\mathbf{e}_{j} \mathbf{e}_{k}^{T}+\mathbf{e}_{k+n} \mathbf{e}_{j+n}^{T}\right) \mathbf{H}^{\nu-1-s} \mathbf{J R H}^{c}\left(\mathbf{e}_{j} \mathbf{e}_{k}^{T}+\mathbf{e}_{k+n} \mathbf{e}_{j+n}^{T}\right) \mathbf{H}^{\mu-1-c} \\
& \mathbf{T}_{2}^{(c)}=-\frac{1}{n} \sum_{s=0}^{m-1} \mathbf{H}^{\nu} \mathbf{J} \mathbf{R} \mathbf{H}^{s}\left(\mathbf{e}_{j} \mathbf{e}_{k}^{T}+\mathbf{e}_{k+n} \mathbf{e}_{j+n}^{T}\right) \mathbf{H}^{m-1-s} \mathbf{J} \mathbf{R} \mathbf{H}^{c}\left(\mathbf{e}_{j} \mathbf{e}_{k}^{T}+\mathbf{e}_{k+n} \mathbf{e}_{j+n}^{T}\right) \mathbf{H}^{\mu-1-c} \\
& \mathbf{T}_{3}^{(c)}=-\frac{1}{n} \sum_{s=0}^{c-1} \mathbf{H}^{\nu} \mathbf{J} \mathbf{R} \mathbf{H}^{s}\left(\mathbf{e}_{j} \mathbf{e}_{k}^{T}+\mathbf{e}_{k+n} \mathbf{e}_{j+n}^{T}\right) \mathbf{H}^{c-1-s}\left(\mathbf{e}_{j} \mathbf{e}_{k}^{T}+\mathbf{e}_{k+n} \mathbf{e}_{j+n}^{T}\right) \mathbf{H}^{\mu-1-c} \\
& \mathbf{T}_{4}^{(c)}=-\sum_{s=1}^{\mu-2-c} \mathbf{H}^{\nu} \mathbf{J} \mathbf{R} \mathbf{H}^{c}\left(\mathbf{e}_{j} \mathbf{e}_{k}^{T}+\mathbf{e}_{k+n} \mathbf{e}_{j+n}^{T}\right) \mathbf{H}^{s}\left(\mathbf{e}_{j} \mathbf{e}_{k}^{T}+\mathbf{e}_{k+n} \mathbf{e}_{j+n}^{T}\right) \mathbf{H}^{\mu-2-c-s}
\end{aligned}
$$


Finally,

$$
\begin{aligned}
& \mathbf{U}_{1}^{(b)}=-\sum_{s=0}^{\nu-1} \mathbf{H}^{s}\left(\mathbf{e}_{j} \mathbf{e}_{k}^{T}+\mathbf{e}_{k+n} \mathbf{e}_{j+n}^{T}\right) \mathbf{H}^{\nu-1-s} \mathbf{J R H}^{b}\left(\mathbf{e}_{j} \mathbf{e}_{k}^{T}+\mathbf{e}_{k+n} \mathbf{e}_{j+n}^{T}\right) \mathbf{H}^{m-1-b} \mathbf{J R} \mathbf{H}^{\mu} \\
& \mathbf{U}_{2}^{(b)}=-\sum_{s=0}^{m-1} \mathbf{H}^{\nu} \mathbf{J} \mathbf{R} \mathbf{H}^{s}\left(\mathbf{e}_{j} \mathbf{e}_{k}^{T}+\mathbf{e}_{k+n} \mathbf{e}_{j+n}^{T}\right) \mathbf{H}^{m-1-s} \mathbf{J} \mathbf{R} \mathbf{H}^{b}\left(\mathbf{e}_{j} \mathbf{e}_{k}^{T}+\mathbf{e}_{k+n} \mathbf{e}_{j+n}^{T}\right) \mathbf{H}^{m-1-b} \mathbf{J R H}^{\mu} \\
& \mathbf{U}_{3}^{(b)}=-\sum_{s=0}^{b-1} \mathbf{H}^{\nu} \mathbf{J} \mathbf{R} \mathbf{H}^{s}\left(\mathbf{e}_{j} \mathbf{e}_{k}^{T}+\mathbf{e}_{k+n} \mathbf{e}_{j+n}^{T}\right) \mathbf{H}^{b-1-s}\left(\mathbf{e}_{j} \mathbf{e}_{k}^{T}+\mathbf{e}_{k+n} \mathbf{e}_{j+n}^{T}\right) \mathbf{H}^{m-1-b} \mathbf{J R} \mathbf{H}^{\mu} \\
& \mathbf{U}_{4}^{(b)}=-\sum_{s=0}^{b-1} \mathbf{H}^{\nu} \mathbf{J} \mathbf{R} \mathbf{H}^{b}\left(\mathbf{e}_{j} \mathbf{e}_{k}^{T}+\mathbf{e}_{k+n} \mathbf{e}_{j+n}^{T}\right) \mathbf{H}^{s}\left(\mathbf{e}_{j} \mathbf{e}_{k}^{T}+\mathbf{e}_{k+n} \mathbf{e}_{j+n}^{T}\right) \mathbf{H}^{m-2-b} \mathbf{J R H}^{\mu} \\
& \mathbf{U}_{5}^{(b)}=-\sum_{s=0}^{m-1} \mathbf{H}^{\nu} \mathbf{J} \mathbf{R} \mathbf{H}^{b}\left(\mathbf{e}_{j} \mathbf{e}_{k}^{T}+\mathbf{e}_{k+n} \mathbf{e}_{j+n}^{T}\right) \mathbf{H}^{m-1-b} \mathbf{J} \mathbf{R} \mathbf{H}^{s}\left(\mathbf{e}_{j} \mathbf{e}_{k}^{T}+\mathbf{e}_{k+n} \mathbf{e}_{j+n}^{T}\right) \mathbf{H}^{m-1-s} \mathbf{J R H} \mathbf{H}^{\mu} \\
& \mathbf{U}_{6}^{(b)}=-\sum_{s=0}^{\mu-1} \mathbf{H}^{\nu} \mathbf{J R} \mathbf{H}^{b}\left(\mathbf{e}_{j} \mathbf{e}_{k}^{T}+\mathbf{e}_{k+n} \mathbf{e}_{j+n}^{T}\right) \mathbf{H}^{m-1-b} \mathbf{J R} \mathbf{H}^{s}\left(\mathbf{e}_{j} \mathbf{e}_{k}^{T}+\mathbf{e}_{k+n} \mathbf{e}_{j+n}^{T}\right) \mathbf{H}^{\mu-1-s} .
\end{aligned}
$$

Note that for any matrices $\mathbf{A}$ and $\mathbf{B}$ we have

$$
\left|[\mathbf{A B}]_{j k}\right| \leq\left\|\mathbf{e}_{k}^{T} \mathbf{A}\right\|_{2}\left\|\mathbf{A} \mathbf{e}_{j}\right\|_{2} .
$$

Applying Hölder's inequality, we get, for $\alpha=1$ or $\alpha=3$

$$
\mathbf{E}\left|X_{j k}\right|^{\alpha}\left|\left[\mathbf{P}^{(a)}\right]_{k j}\right| \leq \mathbf{E}^{\frac{\alpha}{4}}\left|X_{j k}\right|^{4} \mathbf{E}^{\frac{4-\alpha}{4}}\left|\left[\mathbf{P}^{(a)}\right]_{k j}\right|^{\frac{4}{4-\alpha}} .
$$

We may use now inequality (5.47) and Lemma 5.2 to obtain the bound, for $\alpha=1$ or $\alpha=3$

$$
\frac{1}{n^{\frac{5}{2}}} \sum_{j, k=1}^{n} \mathbf{E}\left|X_{j k}\right|^{\alpha}\left|\left[\mathbf{P}_{l}^{(a)}\right]_{k j}\right| \leq \frac{C\left(1+v^{2}\right)}{\sqrt{n} v^{3}}
$$

Similar we get

$$
\frac{1}{n^{\frac{5}{2}}} \sum_{j, k=1}^{n} \mathbf{E}\left|X_{j k}\right|^{3}\left|\left[\mathbf{T}_{l}^{(c)}\right]_{k j}\right| \leq \frac{C(1+v)}{\sqrt{n} v^{3}}
$$

and

$$
\frac{1}{n^{\frac{5}{2}}} \sum_{j, k=1}^{n} \mathbf{E}\left|X_{j k}\right|^{3}\left|\left[\mathbf{U}_{l}^{(b)}\right]_{k j}\right| \leq \frac{C(1+v)}{\sqrt{n} v^{3}}
$$

Inequalities (5.49)-(5.51) together conclude the proof of the Lemma.

Lemma 5.7. Under conditions of Theorem 1.1 we have, for $\mu, \nu \geq 0$ and for any positive $\eta>0$ that there exists a constant $C(\mu, \nu, \eta)$ depending on $\mu, \nu, \eta$ such that

$$
\sum_{j, k=1}^{n} \mathbf{E} X_{j k}\left[\mathbf{H}^{\nu} \mathbf{J} \mathbf{R} \mathbf{H}^{\mu}\right]_{k l}=\sum_{j, k=1}^{n} \mathbf{E}\left[\frac{\partial\left(\mathbf{H}^{\nu} \mathbf{J R} \mathbf{H}^{\mu}\right)}{\partial X_{j k}}\right]_{k l}+\frac{C \theta(1+v}{\sqrt{n} v^{3}},
$$


where $\theta$ denotes a function that $|\theta| \leq 1$.

Proof. Let $\xi$ be random variable with $\mathbf{E} \xi=0, E \xi^{2}=1$ and let $f(x)$ denote a function which satisfies the following condition $\mathbf{E}|\xi|^{3}\left|f^{\prime \prime}(\theta \xi)\right| \leq \varkappa$. Here $\theta$ denotes a uniformly distributed random variable on $[0,1]$. By Tailor's formula we have

$$
\mathbf{E} \xi f(\xi)=\mathbf{E} f^{\prime}(\xi)-\mathbf{E} \xi f^{\prime \prime}(\theta \xi)+\frac{1}{2} \mathbf{E} \xi^{3} f^{\prime \prime}(\theta \xi),
$$

where $\theta$ denotes a uniformly distributed random variable independent of $\xi$. Applying this formula twice and Hölder's inequality, we get

$$
\begin{array}{r}
\left|\sum_{j, k=1}^{n} \mathbf{E} X_{j k}\left[\mathbf{H}^{\nu} \mathbf{J} \mathbf{R H}{ }^{\mu}\right]_{k j}-\sum_{j, k=1}^{n} \mathbf{E}\left[\frac{\partial\left(\mathbf{H}^{\nu} \mathbf{J} \mathbf{R} \mathbf{H}^{\mu}\right)}{\partial X_{j k}}\right]_{k j}\right| \\
\leq n^{-\frac{3}{2}} \sum_{j, k=1}^{n} \mathbf{E}\left(\left|X_{j k}\right|+\left|X_{j k}\right|^{3}\right)\left|\left[\frac{\partial^{2}\left(\mathbf{H}^{\nu} \mathbf{J} \mathbf{R} \mathbf{H}^{\mu}\right)}{\partial X_{j k}^{2}}\left(\theta_{j k} X_{j k}\right)\right]_{k j}\right|
\end{array}
$$

Applying now the result of Lemma 5.6. we conclude the proof of Lemma.

\section{References}

[1] Alexeev, N.; Götze, F.; Tikhomirov, A. N. On the asymptotic distribution of singular values of power of random matrices., Lithuanian mathematical journal, Vol. 50, No. 2, 2010, pp. 121-132.

[2] Alexeev, N.; Götze, F.; Tikhomirov, A. N. On the singular spectrum of powers and products of random matrices, Doklady mathematics, vol. 82, N 1, 2010, pp.505-507.

[3] Banica, T. Belinschi, S. Capitaine, M. and Collins B. Free Bessel Laws Preprint. arXiv:0710.5931

[4] Conway, John B. Functions of one complex variable I.

Springer-Verlag, Berlin 1995 - 2nd ed., 316 pp.

[5] Girko, V. L. Spectral theory of random matrices. (Russian) Uspekhi Mat. Nauk 40 (1985), no. 1(241), 67-106.

[6] Oravecz F. On the powers of Voiculescu's circular element. Studia Math. 145 (2001), no. $1,85-95$.

[7] Mingo, J. A. and Speicher, R. Sharp Bounds for Sums Associated to Graphs of Matrices Preprint. arXiv:0909.4277 